\title{
Double initial and caustic curves in diametrically compressed transparent discs - Application to the contact length
}

\author{
Christos F. Markides \\ National Technical University of Athens, School of Applied Mathematical and Physical Sciences, Department of Mechanics, \\ 5 Heroes of Polytechnion Avenue, Theocaris Bld., Zografou Campus, 15773 Athens, Greece \\ markidib@mail.ntua.gr
}

\begin{abstract}
General formulae for double initial and caustic curves (reflected and transmitted) are obtained in the case of smooth contact of two cylindrical elastic bodies of arbitrary radii. Namely, based on the method of reflected and transmitted caustics, the conditions for the development of double initial and contact caustic curves are established as functions of six independent parameters, while easy-to-use closed-form expressions are given for obtaining the contact length. An experimental protocol is then implemented in the case a thin cylindrical transparent disc is compressed between the jaws of the International Society for Rock Mechanics suggested device for the execution of the Brazilian-disc test. The experimental method of caustics can provide the contact length quite accurately, even in the case of double curves which seem that are not always a consequence of a wide contact region.
\end{abstract}

KEYwORDs. Double initial and caustic curves; Compressed discs; Contact length

\section{open 0 Access}

Citation: Markides, Ch. F., Double initial and caustic curves in diametrically compressed transparent discs - Application to the contact length, Frattura ed Integrità Strutturale, 50 (2019) 451-470.

Received: 12.01 .2019

Accepted: 28.05.2019 Published: 01.10.2019

Copyright: (C) 2019 This is an open access article under the terms of the CC-BY 4.0, which permits unrestricted use, distribution, and reproduction in any medium, provided the original author and source are credited.

\section{INTRODUCTION}

$\mathrm{T}$ he experimental method of caustics is now a more than fifty years old method. It is a powerful technique for the study of various issues related to the mechanical behaviour of elastic bodies. It is based on the high sensitivity of light in the slightest change taking place in the surfaces as well as within the thickness of an elastic plate. At first, in the case of an opaque highly polished elastic plate, recording the path of the impinging light after reflection on its distorted surface, significant results can be drawn regarding the response on externally applied loads and constrains in conjunction with the existence or not of geometrical discontinuities. The same is true in the case of a transparent highly polished elastic plate where one has to record the paths of the impinging light after reflection on both (front and rear) surfaces of the plate. In the case of the plane elastic problem the method was first introduced by Manogg [1-4] for the case of Transmitted Caustics and then it was broadened by Theocaris $[5,6]$ who included the method of Reflected Caustics, to be used soon after on numerus applications [7] keeping used uninterruptedly up to nowadays. In this context, a wide variety of problems has been confronted by the method of caustics, as, the description of the stress intensity in 
elastostatic problems [8-10], the mechanical behaviour and crack propagation under dynamic loading conditions [11-17], the measurement of strain concentration in plasticity problems [18], the determination of the J-integral [19], the determination of stress-optical as well as elastic constants of materials [20-22], the location of the crack-tip position [23], the description of load distribution and the definition of the contact length in contact problems [24-29]. What is more, the method has been successfully applied to a wide range of materials, including birefringent ones [30], rock-like [31] and anisotropic ones [32-35], viscoelastic ones [36] and even composite [37] or graded ones [38]. In parallel, several studies have concerned with the improvement of the method of caustics [39-43] while an experiment on the method of Caustics for educational purposes was recently proposed by Younis [44].

In the present study, further to a work of Theocaris and Stassinakis [26], general formulae for double initial curves and their corresponding reflected or/and transmitted caustic curves are provided in the case two cylindrical elastic bodies of arbitrary radii are compressed against each other in the absence of friction. In this direction and under the usual assumptions made in the method of caustics, the conditions for the development of double initial and contact caustic curves are established. As it is seen, the generation or not of double initial and caustic curves depends on six independent parameters which are related to the material properties, the relative dimensions of the two bodies in contact, as well as to the characteristics of the optical set-up. In light of those formulae, expressions for the contact length given in [26] are revisited and easy-to-use closed-form expressions are given for obtaining the contact length based on the well-known Muskhelishvili's solution for the contact problem [45] particularised in [46]. Then an experimental protocol was implemented concerning a divergent laser light beam incident on a thin cylindrical specimen made of a transparent optically isotropic, linearly elastic material, squeezed between the curved jaws of the International Society for Rock Mechanics (ISRM) suggested device for the standardized Brazilian-disc test [47]. As it is shown, the experimental method of caustics can provide the contact length even in the case of double caustic curves (either reflected or transmitted) which seem that can occur not only in the case of wide contact regions, but also in the case of relatively small ones, under certain conditions concerning, among others, the optical set-up, thus satisfying the small contact length assumption in the theoretic solution employed.

\section{THEORETICAL CONSIDERATIONS}

\section{The contact problem}

$\mathrm{L}$ et a circular disc of radius $R$ and thickness $t$ be in equilibrium upon compressed against a curved jaw of arbitrary radius $R_{J}\left(R \leq R_{J}<\infty\right)$, by an overall load $P_{\text {frame. }}$. Assuming that both the disc and jaw are made of homogeneous, isotropic and linearly elastic materials and their cross-sections lie in the $\zeta=x+\mathrm{i} y=r \mathrm{e}^{\mathrm{i} \theta}$ plane, the expressions for Muskhelishvili's complex potential $\Phi(\zeta)$ for the disc, and the corresponding half contact length $\ell$ realized between the disc and the jaw, read in the $O x y$ coordinate system (Fig.1) as [45, 46]:

$$
\Phi(\zeta)=\frac{1-\varrho}{2 K R}\left(\sqrt{\ell^{2}-\zeta^{2}}+\mathrm{i} \zeta\right), \quad \ell=\sqrt{\frac{2 K R P_{\text {frame }}}{\pi t(1-\varrho)}}, \quad K=\frac{x+1}{4 \mu}+\frac{x_{J}+1}{4 \mu_{J}}
$$

In Eqs. (1), $\varrho=R / R_{J}, x$ is Muskhelishvili's constant, equaling $(3-v) /(1+v)$ for plane strain and $(3-4 v)$ for plane stress, and $\mu=E /[2(1+\nu)]$ is the shear modulus (with $E$ and $\nu$ denoting Young's modulus and Poisson's ratio, respectively).

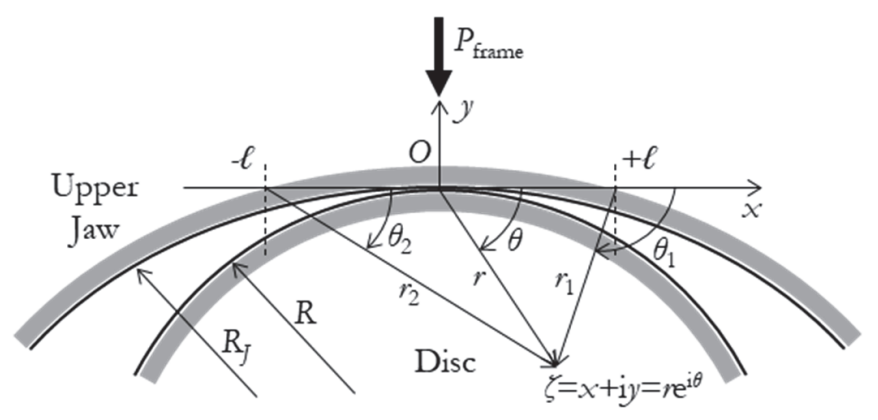

Figure 1: The contact region $(-\ell, \ell)$ and the definition of symbols. 
The general formulae for the contact initial curves on the disc's front and rear faces

Consider the ordinary set-up of the experimental method of caustics shown in Fig.2. Red light, emitted from a He-Ne laser and refined and broadened by a pin-hole, impinges normally, after passing through two lenses $\mathrm{L}_{1}$ and $\mathrm{L}_{2}$ and a semireflector, on the loaded disc. Assuming that the disc is transparent and has two well-polished faces, incident light will be reflected from both the front and rear faces of the disc and it will be transmitted through it as well. Then light reflected and transmitted from points on the disc faces of severe distortion, when received on two screens placed parallel to the disc, at the front and the rear of it, at distances $Z_{o, f}$ and $Z_{o, t}$ from its middle section respectively, forms the so-called reflected and transmitted caustics, which provide significant information about various characteristics about the elastic equilibrium of the disc.

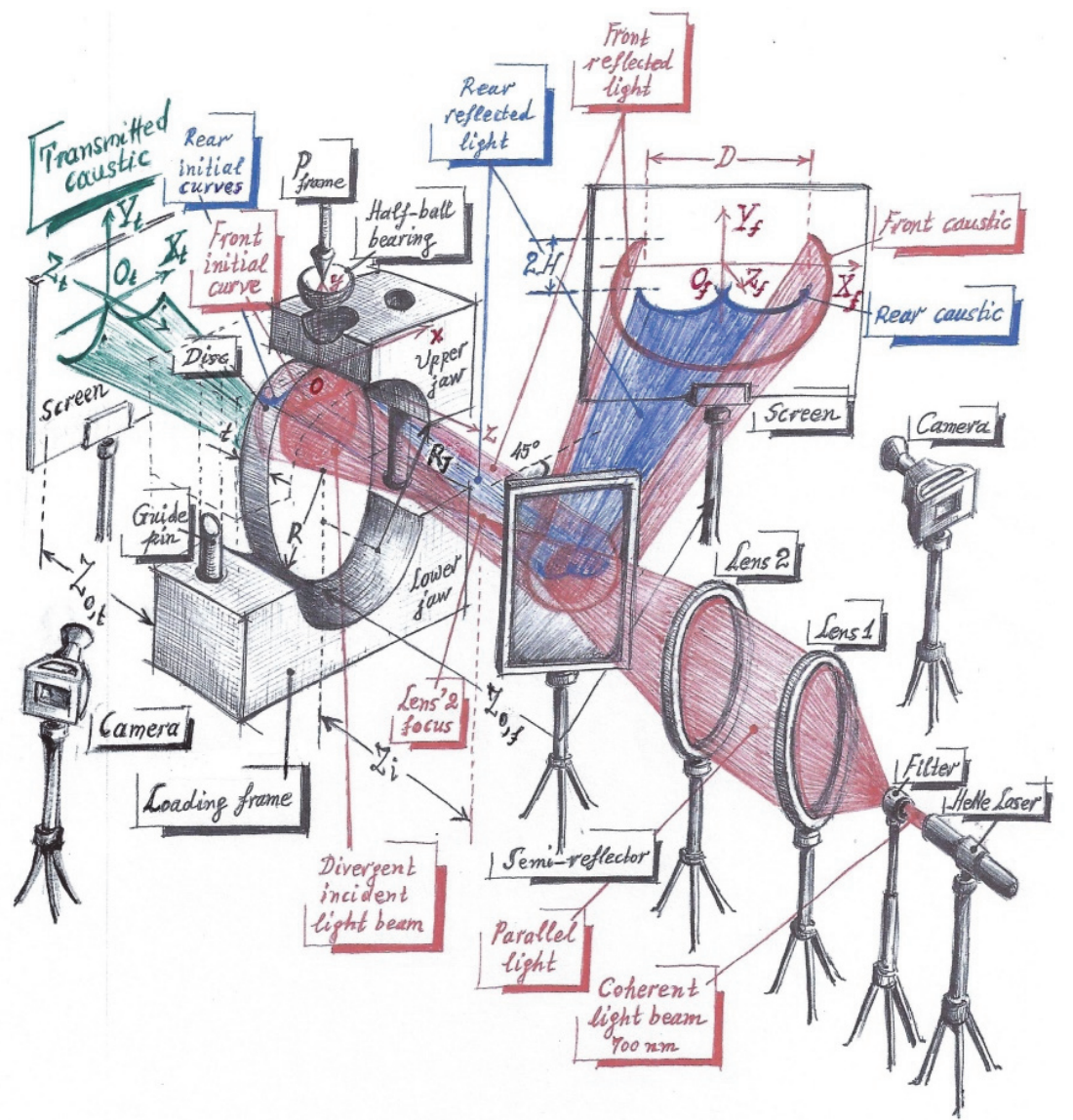

Figure 2: The set-up of the experimental method of reflected and transmitted caustics.

In this context, considering the coordinate systems $O_{f} X_{f} Y_{f} Z_{f}$, and $O_{t} X_{t} Y_{t} Z_{t}$ on the front and the rear screens respectively (so that $X_{f} Z_{f}$ - and $X_{t} Z_{t}$-planes be parallel to $x_{z}$-plane, see Fig.2), assuming that the material of the disc is optically isotropic and that its thickness $t$ is negligible with respect to the distances $Z_{0, f}$ and $Z_{0, t}$ the parametric equations of reflected and transmitted caustics read as [26]:

$$
X_{f, r, t}=\lambda_{m, f, r, t} x-4 C_{f, r, t} \mathfrak{R} \Phi^{\prime}(\zeta), \quad Y_{f, r, t}=\lambda_{m, f, r, t} y+4 C_{f, r, t} \mathfrak{J} \Phi^{\prime}(\zeta)
$$

In Eqs. (2), indexes $f$ and $r$ refer to light reflections from the front and the rear disc's faces while $t$ refers to transmitted light; $\lambda_{m, f, r}$, denotes the respective magnification factor for reflected and transmitted caustics, given as:

$$
\lambda_{m, f}=\lambda_{m, r}=\frac{Z_{o, f} \pm Z_{i}}{Z_{i}}, \quad \lambda_{m, t}=\frac{Z_{o, t} \pm Z_{i}}{Z_{i}} \quad\left(Z_{o, t}>Z_{i}\right)
$$

with $Z_{i}$ being the distance between the focus point of lens $\mathrm{L}_{2}$ and the disc's middle section (Fig.2) and + or - sign indicating a divergent or convergent incident light beam; when lens $\mathrm{L}_{2}$ is missing, $\lambda_{m, t}=\lambda_{m, 1}=\lambda_{m, t}=1$. Moreover: 


$$
C_{f}=-Z_{o, f} t c_{f}, \quad C_{r}=-2 Z_{o, f} t c_{r}, \quad C_{t}=-Z_{o, t} t c_{t}
$$

where $c_{f, r, t}$ are the so-called stress-optical constants defined as:

$$
c_{f}=\frac{\nu}{E}, \quad c_{r}=-k_{c} c_{f}, \quad c_{t}=c_{r}+\frac{\eta_{0}}{2} c_{f}
$$

with $k>0$ being obtained by the interferometric method and $\eta_{0}$ denoting the air-refraction index, equaling approximately zero. Finally, $\mathfrak{R}$ is the real and $\mathfrak{I}$ the imaginary part, while prime indicates first derivative. In turn, zeroing of the Jacobian of the transformation of Eqs. (2), yields the radii of the so-called initial curves, i.e., the loci of points on the disc faces providing upon illumination the caustics, by solving the equation:

$$
\frac{4 C_{f, r, t}}{\lambda_{m, f, r, t}}\left|\Phi^{\prime \prime}(\zeta)\right|= \pm 1
$$

where double prime denotes the second derivative. From Eq.(6) it is clear that there are three kinds of initial curves, one on the front face of the disc, providing the reflected caustics from the front face of the disc, and two on the rear face of the disc providing the reflected and transmitted caustics from the rear face of the disc. Substituting in Eq. (6) for $\Phi(\zeta)$ from Eqs.(1), and using the transformations $\zeta-\ell=r_{1} \mathrm{e}^{\mathrm{i} \theta_{1}}, \zeta+\ell=r_{2} \mathrm{e}^{\mathrm{i} \theta_{2}}, \theta, \theta_{1}, \theta_{2} \epsilon[0,-\pi]$ (Fig.1), the radii $r_{0, f, r, t}$ of the initial curves, the one on the front $\left(r_{0, f}\right)$ and the two $\left(r_{0, r}\right.$ and $\left.r_{0, t}\right)$ on the rear face of the disc, are obtained as:

$$
r_{o, f, r, t}^{ \pm}=\ell \sqrt{\cos 2 \theta \pm \sqrt{\left(2\left|\frac{C_{f, r, t}}{\lambda_{m, f, r, t}}\right| \frac{1-\varrho}{K R \ell}\right)^{4 / 3}-\sin ^{2} 2 \theta}}
$$

In Eq.(7), the absolute value of the ratio $C_{f, f, t} / \lambda_{m, f, r, t}$ has been considered to avoid the double sign \pm on the right-hand side of Eq.(6); that absolute value suffices, also, the demand $r_{1}$ and $r$ be real positive numbers as well as the fact that

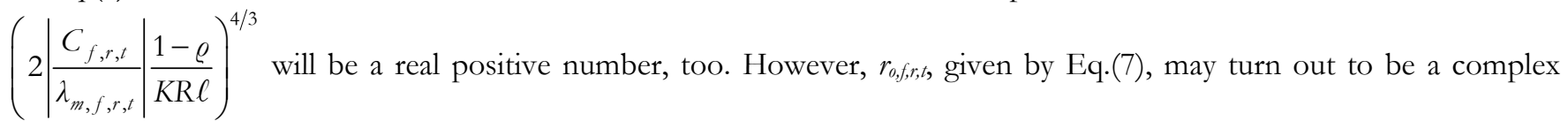
number, something that should be excluded from the solution, keeping only the real values for $r_{0, f, r, t}$. In this context, the double sign before the internal square root, in complete correspondence with the superscript \pm on the left-hand side of Eq.(7), ensures that all possible real values for $r_{0, f, r, t}$ will be taken into account.

Namely, if $\forall \theta \epsilon[0,-\pi],\left(2\left|\frac{C_{f, r, t}}{\lambda_{m, f, r, t}}\right| \frac{1-\varrho}{K R \ell}\right)^{4 / 3} \geq \sin ^{2} 2 \theta$, or simpler if $2\left|\frac{C_{f, r, t}}{\lambda_{m, f, r, t}}\right| \frac{1-\varrho}{K R \ell} \geq 1$, then $\sqrt{\left(2\left|\frac{C_{f, r, t}}{\lambda_{m, f, r, t}}\right| \frac{1-\varrho}{K R \ell}\right)^{4 / 3}-\sin ^{2} 2 \theta}$ is a real positive quantity $\forall \theta \epsilon[0,-\pi]$ and the value $r_{o, f, r, t}^{+}$for the plus sign before the internal square root in Eq.(7) should be chosen for $r_{o, f, r, t}$, rejecting the value $r_{o, f, r, t}^{-}$as a purely imaginary one (see Fig.4b, where it is seen that $r_{o, f, r, t}^{-}$is lying completely in the imaginary plane). That is the classic case of a single initial (and in turn caustic) curve, sketched in Fig.3c (for the front initial curve). In Fig.3a, the variation of $\cos 2 \theta=\sqrt{1-\sin ^{2} 2 \theta}$ is also shown as the lower accepted limiting value for $\sqrt{\left(2\left|\frac{C_{f, r, t}}{\lambda_{m, f, r, t}}\right| \frac{1-\varrho}{K R \ell}\right)^{4 / 3}-\sin ^{2} 2 \theta}$ being a real quantity; beyond that limit, $\sqrt{\left(2\left|\frac{C_{f, r, t}}{\lambda_{m, f, r, t}}\right| \frac{1-\varrho}{K R \ell}\right)^{4 / 3}-\sin ^{2} 2 \theta}$ and $r_{0, f, r, t}$ partly "pass" in the imaginary plane (Fig.4) and they must be accordingly rejected as it is shown next for a double initial curve.

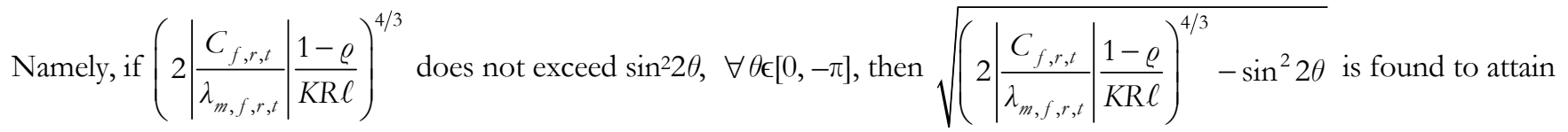




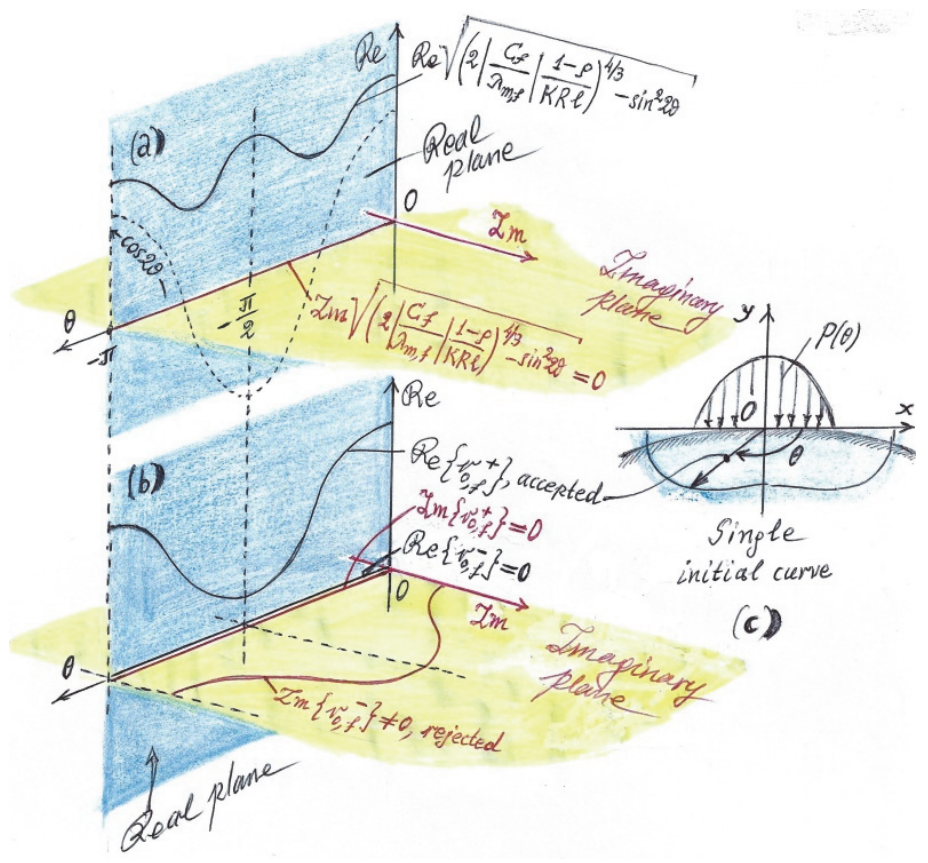

Figure 3: (a) The case the internal square root in Eq.(7) assumes real positive values; (b) The relative accepted radius of the initial curve; (c) The corresponding single initial curve.

either real or purely imaginary values, depending on the interval $\theta$ takes values in. Namely, considering the critical angle (measured from $x$-axis in the clockwise direction):

$$
\theta_{o, f, r, t}=\frac{1}{2} \operatorname{Arcsin}\left(2\left|\frac{C_{f, r, t}}{\lambda_{m, f, r, t}}\right| \frac{1-\varrho}{K R \ell}\right)^{2 / 3}
$$

the quantity $\sqrt{\left(2\left|\frac{C_{f, r, t}}{\lambda_{m, f, r, t}}\right| \frac{1-\varrho}{K R \ell}\right)^{4 / 3}-\sin ^{2} 2 \theta}$ assumes real positive values for $\theta \epsilon\left[0,-\theta_{0, f, r, t}\right],\left(-\pi / 2+\theta_{0, f, r, t,}-\pi / 2-\theta_{0, f, r, t}\right]$ and

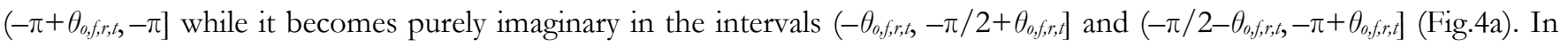
turn, $r_{o, f, r, t}$ (Eq.(7)) assumes complex values in the intervals $\left(-\theta_{0, f, r, t},-\pi / 2+\theta_{0, f, r, t}\right.$ and $\left(-\pi / 2-\theta_{0, f, r, t,}-\pi+\theta_{0, f, r, t}\right]$, purely imaginary ones in the interval $\left(-\pi / 2+\theta_{0, f, r, t,}-\pi / 2-\theta_{0, f, r, t}\right]$ and real positive values in the intervals $\left[0,-\theta_{0, f, r, t}\right]$ and $\left(-\pi+\theta_{0, f, r, t,},-\pi\right]$ (Fig. $\left.4 \mathrm{~b}\right)$. Then in this context, since only a real-valued quantity can stand as the radius $r_{o, f, r, t}$ of the initial curve, it is seen that in that case the initial curve splits into two distinct curves (parts), defined in the intervals $\left[0,-\theta_{0, f, r, t}\right]$ and $\left(-\pi+\theta_{0, f, r, t,}, \pi\right]$, respectively, where the imaginary parts of the aforementioned quantities are zero. These two parts are located around the end points of the contact length, symmetrically with respect to $y$-axis (Fig.4c). Namely, the right part is located around the point $(+\ell, 0)$ and is defined in the interval $\left[0,-\theta_{0, f, r, t}\right]$ while the left one is located around the point $(-\ell, 0)$ and is defined in the interval $\left(-\pi+\theta_{0, f, r, t,}, \pi\right]$. In addition, it should be noticed that both $r_{o, f, r, t}^{+}$and $r_{o, f, r, t}^{-}$are required to describe the radius $r_{0, f, r, t}$ of either part. Actually, considering for example the right part, it can be seen that it consists of two branches, viz., the outermost one and the innermost one, with respect to the origin. The radius of the outermost branch is described by the $r_{o, f, r, t}^{+}$-value while that of the innermost one by the $r_{o, f, r, t}^{-}$-value, and as $\theta$ varies in the clockwise direction within the interval $\left[0,-\theta_{0, f, r, t}\right]$ the two branches are formed simultaneously, tracing the right part of the initial curve following opposite directions to eventually meet at their single point in common. Namely, the outermost branch of the right part starts (for $\theta=0)$ from a point $\zeta$ of $x$-axis on the right side of the point $(+\ell, 0)$ while its innermost one starts simultaneously (for $\theta=0$ ) from a point $\zeta$ of $x$-axis on the left side of the point $(+\ell, 0)$. Then, to each $\theta$-value between 0 and $-\theta_{o, f, r, t}$ they correspond two points, as the pinpoints of the vectors $\boldsymbol{r}_{o, f, r, t}^{+}$and $\boldsymbol{r}_{o, f, r, t}^{-}$, one on outermost branch and the second one on the inner- 
most part, respectively. These two points are all the time along the same line (defined by the $\theta$-direction), in other words $\boldsymbol{r}_{o, f, r, t}^{+}$and $\boldsymbol{r}_{o, f, r, t}^{-}$are all the time collinear, but of different in general measure. Eventually, $\boldsymbol{r}_{o, f, r, t}^{+}$and $\boldsymbol{r}_{o, f, r, t}^{-}$mutually met (for $\left.\theta=\theta_{o, f, r, t}\right)$ at the point $\zeta_{o, f, r, t}=r_{o, f, r, t}^{+} \mathrm{e}^{\mathrm{i} \theta_{o, f, r, t}}=r_{o, f, r, t}^{-} \mathrm{e}^{\mathrm{i} \theta_{\theta, f, r, t}}$ of the two branches in common, thus completing the formation of the right part of the double initial curve; regarding the previous notation, $\zeta_{o, f, r, t}=x_{o, f, r, t}+\mathrm{i} y_{o, f, r, t}=r_{o, f, r, t}^{ \pm} \mathrm{e}^{\mathrm{i} \theta}$ is to denote the point $\zeta$ on the initial curve. The left part of the initial curve, i.e., that around the point $(-\ell, 0)$, is formed in a completely analogous manner. In this context, the parametric equations of the double caustic are defined in the intervals $\theta \in\left[0,-\theta_{0, f, r, t}\right]$ and $\theta \in\left(-\pi+\theta_{0 . f, r, t,}-\pi\right]$ as:

$$
x_{o, f, r, t}=r_{o, f, r, t}^{ \pm} \cos \theta, \quad y_{o, f, r, t}=r_{o, f, r, t}^{ \pm} \sin \theta
$$

Obviously, the formulae of Eqs. (9) for a double initial curve are general by means that can also provide the case of a single curve, the radius $r_{o, f, r, t}$ of which is completely defined, $\forall \theta \in[0,-\pi]$ by just considering the value $r_{o, f, r, t}^{+}$only.

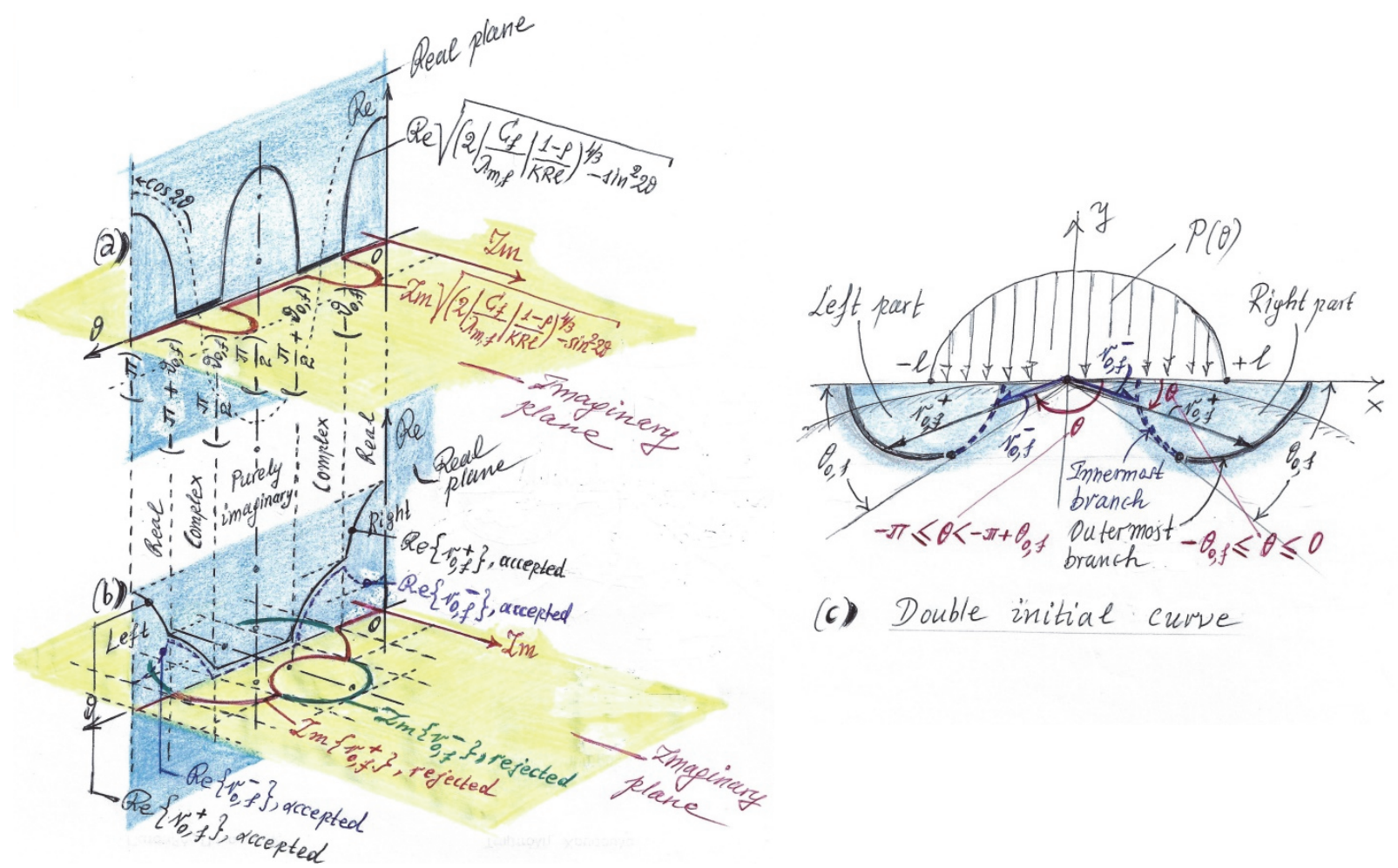

Figure 4: (a) The case the internal square root in Eq.(7) attains imaginary values in some $\theta$-intervals; (b) The relative values for the radius of the initial curve; (c) The corresponding double initial curve with the left and right part, and the two branches of each part.

\section{The role of various parameters in the generation of double initial curves}

From the previous paragraph, it is seen that the condition dictating the generation or not of a double initial curve is:

$$
F_{f, r, t}=2\left|\frac{C_{f, r, t}}{\lambda_{m, f, r, t}}\right| \frac{1-\varrho}{K R \ell}<1
$$

with $F_{f, r, t}$ a dimensionless quantity. Assuming that the disc's dimensions $(R, t)$ are kept fixed, that its material's Poisson ratio $v$ as well as the jaw's material elastic properties $\left(E_{J}, v_{J}\right)$ have a negligible influence on the generation of double curves, and that an average value for $k$ (in the second of Eqs. (5)) has once and for all been chosen for a reasonably bounded range of materials checked, Eq.(10) is written respectively for the three initial curves, the one on the front and the two on the rear disc's faces, as: 


$$
\begin{aligned}
& F_{f}\left(E, P_{\text {frame }}, R_{J}, Z_{o, f}, Z_{i}\right)=2\left|\frac{C_{f}\left(E, Z_{o, f}\right)}{\lambda_{m, f}\left(Z_{o, f}, Z_{i}\right)}\right| \frac{1-\varrho\left(R_{J}\right)}{K(E) R \ell\left(P_{\text {frame }}, R_{J}, E\right)}<1 \\
& F_{r}\left(E, P_{\text {frame }}, R_{J}, Z_{o, f}, Z_{i}\right)=2\left|\frac{C_{r}\left(E, Z_{o, f}\right)}{\lambda_{m, f}\left(Z_{o, f}, Z_{i}\right)}\right| \frac{1-\varrho\left(R_{J}\right)}{K(E) R \ell\left(P_{\text {frame }}, R_{J}, E\right)}<1 \\
& F_{t}\left(E, P_{\text {frame }}, R_{J}, Z_{o, t}, Z_{i}\right)=2\left|\frac{C_{t}\left(E, Z_{o, t}\right)}{\lambda_{m, t}\left(Z_{o, t}, Z_{i}\right)}\right| \frac{1-\varrho\left(R_{J}\right)}{K(E) R \ell\left(P_{\text {frame }}, R_{J}, E\right)}<1
\end{aligned}
$$

where

$$
\begin{aligned}
& C_{f}\left(E, Z_{o, f}\right)=-Z_{o, f} t c_{f}(E), \quad C_{r}\left(E, Z_{o, f}\right)=-2 Z_{o, f} t c_{r}(E), \quad C_{t}\left(E, Z_{o, t}\right)=-Z_{o, t} t c_{t}(E) \\
& c_{f}(E)=\frac{\nu}{E}, \quad c_{r}(E)=-k c_{f}(E), \quad c_{t}(E)=c_{r}(E)+\frac{\eta_{o}}{2} c_{f}(E) \\
& \lambda_{m, f}\left(Z_{o, f}, Z_{i}\right)=\lambda_{m, r}\left(Z_{o, f}, Z_{i}\right)=\frac{Z_{o, f} \pm Z_{i}}{Z_{i}}, \quad \lambda_{m, t}\left(Z_{o, t}, Z_{i}\right)=\frac{Z_{o, t} \pm Z_{i}}{Z_{i}} \\
& \varrho\left(R_{J}\right)=\frac{R}{R_{J}}, \quad K(E)=\frac{(x+1)(1+v)}{2 \mu E}+\frac{x_{J}+1}{4 \mu_{J}}, \quad \ell\left(P_{\text {frame }}, R_{J}, E\right)=\sqrt{\frac{2 K(E) R P_{\text {frame }}}{\pi t\left[1-\varrho\left(R_{J}\right)\right]}}
\end{aligned}
$$

Under the above assumptions, it is seen that six independent parameters, namely, $E, P_{\text {frame, }}, R_{J}, Z_{o, f}, Z_{o, t}$ and $Z_{i}$, entering Eqs. (11), influence the development of a double initial curve. The degree of their influence is shown in Fig.5, where the quantities $F_{f}, F_{r}$, and $F_{t}$ are plotted (in juxtaposition) against each one of these six parameters. To draw this figure, a reference set-up has been considered as a basis, namely, that concerning a disc of $R=5 \mathrm{~cm}, t=1 \mathrm{~cm}$, made of Poly-MethylMeth-Acrylate (PMMA) $(\boldsymbol{E}=3.20 \mathrm{GPa}, \boldsymbol{\nu}=0.38)$ with $k=3.12$ [48], squeezed between the ISRM's curved steel jaws $\left(\boldsymbol{R}_{\boldsymbol{J}}=1.5 \mathrm{R}, E_{J}=210 \mathrm{GPa}, v_{J}=0.30\right)$ for the implementation of the Brazilian-disc test [47], by an overall load $\boldsymbol{P}_{\text {frame }}=15 \mathrm{kN}$. The disc was under plane stress- and the jaw under plane strain-conditions. In addition, it was assumed that $\boldsymbol{Z}_{\boldsymbol{o}, \boldsymbol{f}}=1.5 \mathrm{~m}$, $Z_{o, t}=0.7 \mathrm{~m}$ and $Z_{i}=1.0 \mathrm{~m}$, with the second lens' focus point located before the disc, i.e., a divergent incident light beam was considered ( $E, P_{\text {frame }}, R_{J}, Z_{o, f}, Z_{o, t}$ and $Z_{i}$ were put in bold for clarity). Then, in each of the plots from Fig.5(a-f), keeping four out of the five different independent parameters appearing each time in $F_{f}, F_{r}$ and $F_{t}$, fixed and letting the fifth one vary within a reasonable interval, the variations of $F_{f}$ (red color), $F_{r}$ (blue color) and $F_{t}$ (green color) are plotted.

In this context, keeping $P_{\text {frame }}, R_{J}, Z_{o, f}$, and $Z_{i}$ constant, equal to the respective values of the reference set-up, Fig.5a shows the variations of $F_{f}, F_{r}$ and $F_{t}$ against the modulus of elasticity $E$; the $F_{f, r, t}=1$ line is also shown as the critical value below which a double initial curve appears. Thus, for an $E$ less than $1.25 \mathrm{GPa}$, then $F_{f}$ is less than 1 and a double front initial curve occurs. For an $E$ less than $0.4 \mathrm{GPa}$, then $F_{t}$ is less than 1 and a double rear transmission initial curve is generated. Regarding the rear reflective initial curve, it is seen that only in the case of a material with very high compliance, i.e., with an $E$ less than $0.02 \mathrm{GPa}$, a double initial curve appears. In Fig.5b, fixing $E, R_{f}, Z_{o, f}$, and $Z_{i}$, the variation of $F_{f}, F_{r}$ and $F_{t}$ versus $P_{\text {frame }}$ is shown together with the limiting line $F_{f, r}=1$. Starting from the reflective and the transmitting initial curves on the disc's rear face, it is seen that only an extraordinary $P_{\text {frame }}$ could make them split into two parts while a $P_{\text {frame }}$ of a value $38 \mathrm{kN}$ upwards would result in a double front initial curve. In Fig.5c, fixing $E, P_{\text {frame, }}, Z_{o, f}$, and $Z_{i}$, the variation of $F_{f}$, $F_{r}$ and $F_{t}$ is plotted versus $R_{J}$. It is definitely seen, for example, that for the chosen reference set-up values, i.e., for the ISRM's jaw with $R_{f}=1.5 R=0.075 \mathrm{~m}$, a double initial curve cannot happen, as in that case all three $F_{f}, F_{r}$ and $F_{t}$ values are bigger that 1 ; but as $R_{J}$ decreases a double front initial curve appears for $R_{J}=0.067 \mathrm{~m}$, a double transmitting initial curve occurs for $R_{J}=0.06 \mathrm{~m}$ while a double rear reflective curve is generated for $R_{J}=0.054 \mathrm{~m}$. In Fig. $5 \mathrm{~d}$, fixing $E, P_{\text {frame }}, R_{J}, Z_{o, f}$ and $Z_{o, t}$ the variation of $F_{f}, F_{r}$ and $F_{t}$ is plotted versus $Z_{i}$. It is seen that for a value $Z_{i}=0.07 \mathrm{~m}$ downwards a double rear reflective initial curve appears, for a value $Z_{i}=0.19 \mathrm{~m}$ downwards a double transmission initial curve appears while for a value $Z_{i}=0.52 \mathrm{~m}$ downwards a double front initial curve occurs.

In Fig.5e, fixing $E, P_{\text {frame }}, R_{J}$ and $Z_{i}$, the variation of $F_{f}$ and $F_{r}$ is plotted against $Z_{0, f}$. As it is seen, placing the front reference screen at a distance $Z_{o, f}$ less than $0.6 \mathrm{~m}$ from the disc's middle section, results always in a double front initial curve whereas a rather unmaterialized distance $Z_{o, f}$, less than $0.07 \mathrm{~m}$, is required to pump out a rear double reflective initial curve. Finally, in Fig.5f, fixing $E, P_{\text {frame }}, R_{J}$ and $Z_{i}$, the variation of $F_{t}$ is plotted against $Z_{o, t .}$ It is seen that placing the rear reference screen at a distance $Z_{o, t}$ less than $0.18 \mathrm{~m}$ from the disc's middle section, results always in a double rear initial 
transmission curve. From the above results it becomes evident that the generation of a double initial curve is not only the result of an increased externally applied load; it can be also caused by other factors, influencing the experiment, like the material of the specimen and other parameters directly connected with the experimental/optical set-up. And it is concluded that even in the case of relatively small contact lengths, there could be double initial (and thus caustic) curves. Finally, it should be mentioned, that according to the above findings the development of a double rear reflective initial curve is positively more difficult to happen with respect to the two other kinds of initial curves.
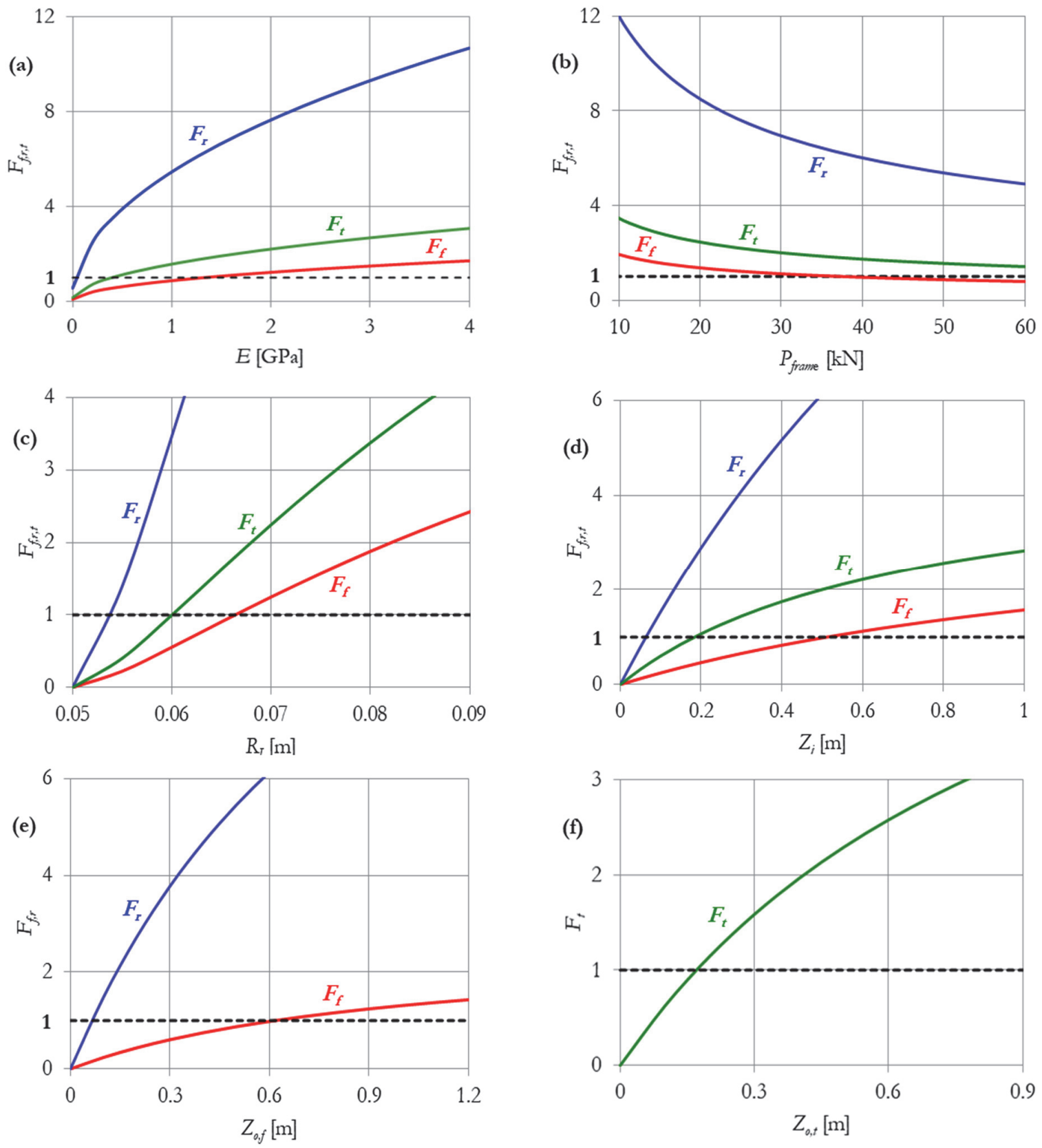

Figure 5: (a) The variation of $F_{f}, F_{r}$ and $F_{t}$ against $E$; (b) The variation of $F_{f}, F_{r}$ and $F_{t}$ against $P_{\text {frame}}$; (c) The variation of $F_{f}, F_{r}$ and $F_{t}$ against $R_{j}$; (d) The variation of $F_{f}, F_{r}$ and $F_{t}$ against $Z_{i}$; (e) The variation of $F_{f}$ and $F_{r}$ against $Z_{o, f}$; (f) The variation of $F_{t}$ against $Z_{o, t}$.

\section{The general formulae for reflected and transmitted contact caustics}

The parametric equations of single or double reflected and transmitted caustics, corresponding to the previously presented single or double initial curves, are obtained by substituting in Eqs. (2) for $\Phi(\zeta)$ from Eqs. (1) and using the relations $\zeta-\ell=r_{1} \mathrm{e}^{\mathrm{i} \theta_{1}}, \zeta+\ell=r_{2} \mathrm{e}^{\mathrm{i} \theta_{2}}, \theta, \theta_{1}, \theta_{2} \in[0,-\pi]$ for $\zeta \equiv \zeta_{o, f, r, t}=r_{o, f, r, t}^{ \pm} \mathrm{e}^{\mathrm{i} \theta}$ on the initial curves $\left(r_{o, f, r, t}^{ \pm}\right.$given by Eq. (7)), as: 


$$
\begin{aligned}
& X_{f, r, t}^{ \pm}=\lambda_{m, f, r, t} r_{o, f, r, t}^{ \pm} \cos \theta-\left(2 \frac{1-\varrho}{K R \ell}\right)^{2 / 3} C_{f, r, t}\left|\frac{\lambda_{m, f, r, t}}{C_{f, r, t}}\right|^{1 / 3} r_{o, f, r, t}^{ \pm} \sin \left(\theta-\frac{\theta_{1, f, r, t}^{ \pm}+\theta_{2, f, r, t}^{ \pm}}{2}\right), \\
& Y_{f, r, t}^{ \pm}=\lambda_{m, f, r, t} r_{o, f, r, t}^{ \pm} \sin \theta+C_{f, r, t}\left[2 \frac{1-\varrho}{K R}-\left(2 \frac{1-\varrho}{K R \ell}\right)^{2 / 3}\left|\frac{\lambda_{m, f, r, t}}{C_{f, r, t}}\right|^{1 / 3} r_{o, f, r, t}^{ \pm} \cos \left(\theta-\frac{\theta_{1, f, r, t}^{ \pm}+\theta_{2, f, r, t}^{ \pm}}{2}\right)\right]
\end{aligned}
$$

In Eqs. (13), $\theta_{j, f, r, t}^{ \pm}, j=1,2$ are defined as (Fig.1):

$$
\theta_{1, f, r, t}^{ \pm}=\operatorname{Arctan} \frac{r_{o, f, r, t}^{ \pm} \sin \theta}{r_{o, f, r, t}^{ \pm} \cos \theta-\ell}, \quad \theta_{2, f, r, t}^{ \pm}=\operatorname{Arctan} \frac{r_{o, f, r, t}^{ \pm} \sin \theta}{r_{o, f, r, t}^{ \pm} \cos \theta+\ell}
$$

(obviously, $\theta_{j, f}^{ \pm} \neq \theta_{j, r}^{ \pm} \neq \theta_{j, t}^{ \pm}$because for each $\theta, r_{o, f}^{ \pm} \neq r_{o, r}^{ \pm} \neq r_{o, t}^{ \pm}$). Eqs. (13) complete the existing formulae of Theocaris and Stassinakis [26], providing all possible cases for reflected and transmitted caustics, viz., either double or single ones. As an example, the above formulae are applied to a hypothetical case resulting, on purpose, in only double curves. Namely, a circular disc of $R=5 \mathrm{~cm}, t=1 \mathrm{~cm}$ is considered, made of PMMA $(E=3.20 \mathrm{GPa}, \nu=0.38)$ and with a $k=2.5\left(2^{\text {nd }}\right.$ of Eqs. (5)). The disc was squeezed between two curved jaws of $R_{J}=1.3 R$, made of steel $\left(E_{J}=210 \mathrm{GPa}, v_{J}=0.30\right)$, by an overall load $P_{\text {frame }}=30 \mathrm{kN}$. The disc was considered under plane stress conditions, while the jaws were considered under plane strain conditions. In addition, it was assumed that $Z_{0, f}=1.0 \mathrm{~m}$ and $Z_{i}=0.2 \mathrm{~m}$ (before the disc-a divergent impinging light beam) and that $Z_{o, t}=0.7 \mathrm{~m}$. The double initial curves, due to Eqs. (9), are plotted for the above data in Fig.6, where the whole contact region is shown. As before, red, blue and green color indicates the front, rear and transmission initial curves. Actually, red, blue and green lines indicate the outermost branches of the left and right parts of the double initial curves, corresponding to the $r_{o, f}^{+}$-value of $r_{o, f}$, while black lines indicate the innermost branches, corresponding to the $r_{o, f}^{-}$-value of $r_{o, f}$.

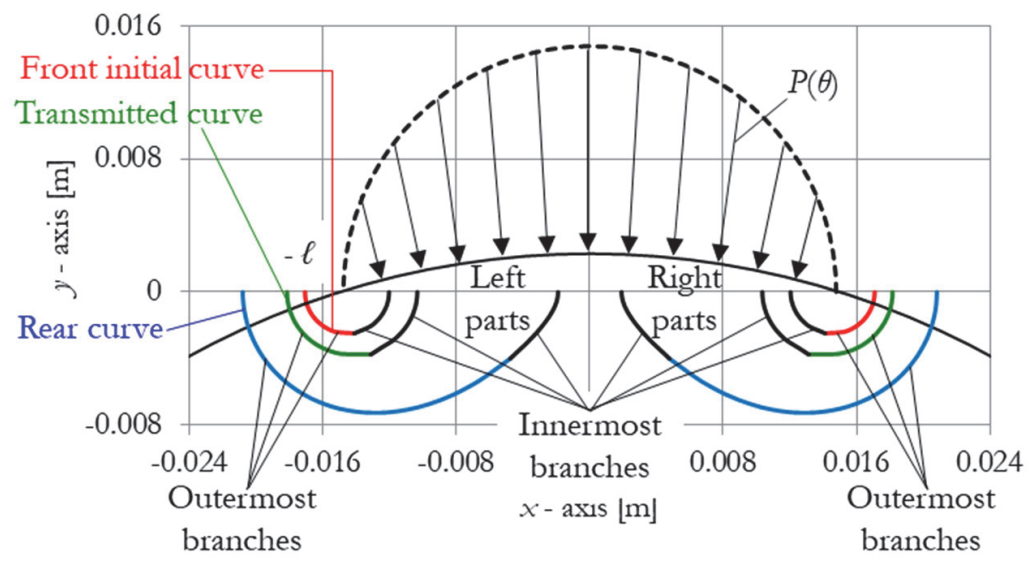

Figure 6: The contact region with the double initial curves

Accordingly, the double caustic curves, due to Eqs. (13), are plotted in Fig.7, following (in complete correspondence between color) the same coloring used for the respective initial curves. In addition, the spans $D_{f, r, t}^{+} D_{-f, r, t}$ of the caustics as well as the elevations $H_{f, r, t}^{+}$and $H_{f, r, t}$, of their end points have been specified in Fig.7, required, as it will be shown next, for the estimation of the contact length.

\section{THE CONTACT LENGTH BY USING THE GENERAL CAUSTICS}

$\mathrm{I}$ $\mathrm{n}$ light of the previous general formulae for the contact caustics, viz., Eqs. (7, 13, 14), Theocaris and Stassinakis' [26] existing formulae for obtaining the contact length, realized between two cylindrical bodies in contact, are here completed by including the case of double caustics. In addition, closed-form formulae are provided for obtaining $\ell$ 
from the span

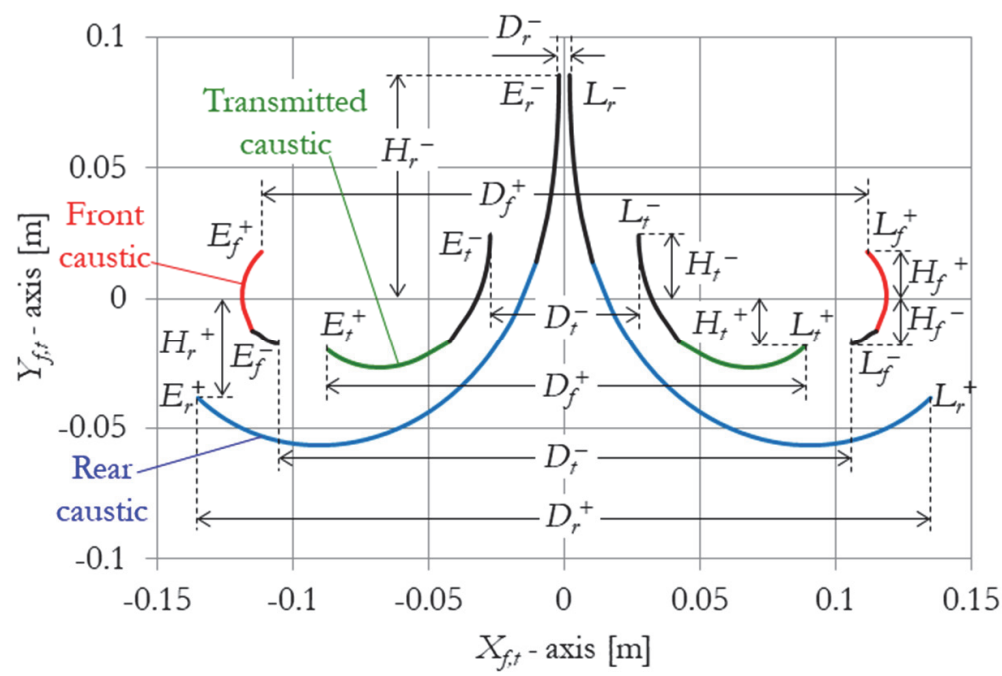

Figure 7: The general case for double reflected and transmitted caustics.

of a double or single caustic while two different ways are proposed to locate $X_{f, t}$-axis on the experimental caustics' photos, necessary for the use of the existing linear formula [26] for obtaining $\ell$ from the elevation of caustic's extreme points.

Obtaining the contact length from the span of the general contact caustic curves

Let us first consider the more general case of double caustics. In this context, the spans of the caustics, i.e., the distances $D_{f, r, t}^{+}$between the outermost ends $E_{f f, r, t}^{+}$and $L_{f, r, t}^{+}$of the caustic curves and the distances $D^{-}{ }_{f, r, t}$ between the innermost ends $E_{f, r, t}$ and $L_{-f, r, t}$ (Fig.7) are defined by the aid of the first of Eqs. (13), as:

$$
\begin{aligned}
& D_{f, r, t}^{+}=2 X_{f, r, t}^{+}(\theta=0)=2 \lambda_{m, f, r, t} \ell \sqrt{1+\left(2\left|\frac{C_{f, r, t}}{\lambda_{m, f, r, t}}\right| \frac{1-\varrho}{K R \ell}\right)^{2 / 3}} \\
& D_{f, r, t}^{-}=2 X_{f, r, t}^{-}(\theta=0)=2\left[\lambda_{m, f, r, t}-\left(2 \frac{1-\varrho}{K R \ell}\right)^{2 / 3} C_{f, r, t}\left|\frac{\lambda_{m, f, r, t}}{C_{f, r, t}}\right|^{1 / 3}\right] \ell \sqrt{1-\left(2\left|\frac{C_{f, r, t}}{\lambda_{m, f, r, t}}\right| \frac{1-\varrho}{K R \ell}\right)^{2 / 3}}
\end{aligned}
$$

The superscripts + and - in the above formulae, are in accordance with the previous discussion and refer to the value $r_{o, f, r, t}^{+}$and $r_{o, f, r, t}^{-}$respectively, describing the radius of the corresponding double initial curve. In this context, the abscissas, $X_{f, r, t}^{+}(\theta=0)$ and $X_{f, r, t}^{+}(\theta=-\pi)$, of $E_{f, r, t}^{+}$and $L_{f, r, t,}^{+}$correspond respectively to the outermost points $r_{o, f, r, t}^{+} \mathrm{e}^{\mathrm{i} 0}$ and $r_{o, f, r, t}^{+} \mathrm{e}^{-\mathrm{i} \pi}$ of the relevant initial curve, while the abscissas, $X_{f, r, t}^{-}(\theta=0)$ and $X_{f, r, t}^{-}(\theta=-\pi)$, of $E_{-f, r, t}$ and $L_{f, r, t,}$ correspond respectively to the innermost points $r_{o, f, r, t}^{-} \mathrm{e}^{\mathrm{i} 0}$ and $r_{o, f, r, t}^{-} \mathrm{e}^{-\mathrm{i} \pi}$ of the same initial curve. What is more, there will be in general two (slightly) different experimental values $\ell^{+}{ }_{D}$ and $\ell^{-}{ }_{D}$ for $\ell$ upon using Eqs. (15) and (16), need to be combined to yield the final experimental $\ell_{D}$, i.e., the half contact length $\ell$ obtained from the span $D$. In this direction, solving Eq. (15) for $\ell$, one can write the expression:

$$
\left(\ell_{D, f, r, t}^{+}\right)^{2}+\left(2\left|\frac{C_{f, r, t}}{\lambda_{m, f, r, t}}\right| \frac{1-\varrho}{K R}\right)^{2 / 3}\left(\ell_{D, f, r, t}^{+}\right)^{4 / 3}-\left(\frac{D_{f, r, t}^{+}}{2 \lambda_{m, f, r, t}}\right)^{2}=0
$$


which agrees with the respective formula derived in [26] (after some minor revisions in [26], see [49], and few modifications introduced here for the sake of generality of the procedure proposed). In Eq.(17), the use of the notation $\ell^{+}{ }_{D, f, r, t}$ instead of simply writing $\ell$, has been made to stress the distinction among $\ell^{+}{ }_{D, f}, \ell^{+} D, r$ and $\ell^{+}{ }_{D, t}$ (corresponding to $D^{+}{ }_{f}, D^{+}{ }_{r}$ and $\left.D^{+}{ }_{t}\right)$ when obtained experimentally. Actually, while in theory all three $\ell^{+}{ }_{D, f}, \ell^{+}{ }_{D, r}$ and $\ell^{+}{ }_{D, t}$ equal $\ell$ given by the second of Eqs. (1) thus fulfilling Eq.(17) by identity, in practice reasonable differences between the theoretically predicted and experimentally measured $D_{f}^{+}, D^{+}{ }_{r}$ and $D^{+}{ }_{t}$ lead inevitably (via Eq.(20), see below) to "experimental" values for $\ell^{+}{ }_{D, f}$, $\ell^{+} D, r$ and $\ell^{+} D, t$, slightly different to each other and from the theoretical $\ell$. Thus, in applying the experimental method of caustics to obtain $\ell_{D}$ there will be in general three different values for $\ell_{D}$, viz., $\ell^{+}{ }_{D, f}, \ell^{+} D, r$ and $\ell^{+} D, t$ which should be properly combined to provide the experimental $\ell_{D}$. Now setting:

$$
\ell_{D, f, r, t}^{+}=\left(m_{f, r, t}^{+}\right)^{3 / 2}
$$

Eq.(17) becomes:

$$
\left(m_{f, r, t}^{+}\right)^{3}+\left(2\left|\frac{C_{f, r, t}}{\lambda_{m, f, r, t}}\right| \frac{1-\varrho}{K R}\right)^{2 / 3}\left(m_{f, r, t}^{+}\right)^{2}-\left(\frac{D_{f, r, t}^{+}}{2 \lambda_{m, f, r, t}}\right)^{2}=0
$$

Eq.(19) admits two complex and one real solution, the latter of which, upon inserted into Eq.(18), yields $\ell^{+}{ }_{D_{f}, f, t}$ as:

$$
\begin{aligned}
& \ell_{D, f, r, t}^{+}=\left\{-\frac{1}{3}\left(2\left|\frac{C_{f, r, t}}{\lambda_{m, f, r, t}}\right| \frac{1-\varrho}{K R}\right)^{2 / 3}+\left\{\frac{1}{2}\left(\frac{D_{f, r, t}^{+}}{2 \lambda_{m, f, r, t}}\right)^{2}-\frac{1}{27}\left(2\left|\frac{C_{f, r, t}}{\lambda_{m, f, r, t}}\right| \frac{1-\varrho}{K R}\right)^{2}+\right.\right. \\
& \left\{-\frac{1}{729}\left(2\left|\frac{C_{f, r, t}}{\lambda_{m, f, r, t}}\right| \frac{1-\varrho}{K R}\right)^{4}+\left[\frac{1}{2}\left(\frac{D_{f, r, t}^{+}}{2 \lambda_{m, f, r, t}}\right)^{2}-\frac{1}{27}\left(2\left|\frac{C_{f, r, t}}{\lambda_{m, f, r, t}}\right| \frac{1-\varrho}{K R}\right)^{2}\right]^{2}\right\}^{1 / 2}+ \\
& \left\{\frac{1}{2}\left(\frac{D_{f, r, t}^{+}}{2 \lambda_{m, f, r, t}}\right)^{2 / 3}-\frac{1}{27}\left(2\left|\frac{C_{f, r, t}}{\lambda_{m, f, r, t}}\right| \frac{1-\varrho}{K R}\right)^{2}-\right. \\
& \left.\left.\left\{-\frac{1}{729}\left(2\left|\frac{C_{f, r, t}}{\lambda_{m, f, r, t}}\right| \frac{1-\varrho}{K R}\right)^{4}+\left[\frac{1}{2}\left(\frac{D_{f, r, t}^{+}}{2 \lambda_{m, f, r, t}}\right)^{2}-\frac{1}{27}\left(2\left|\frac{C_{f, r, t}}{\lambda_{m, f, r, t}}\right| \frac{1-\varrho}{K R}\right)^{2}\right]^{2}\right\}^{1 / 2}\right\}^{1 / 3}\right\}^{3 / 2}
\end{aligned}
$$

According to the method of caustics, measuring the distance $D_{f, r, t}^{+}$on the experimental caustics' photos and introducing that value in Eq.(20), will yield the half contact length $\ell^{+} D_{j, f, t}$ in question. Of course, as it has already been mentioned, reasonable differences between the theoretical and the experimentally measured $D^{+}{ }_{f, r}$, attributed to both theoretical approximations and experimental errors, will lead (via Eq.(20)) to slightly different values $\ell^{+}{ }_{D, f} \ell^{+}{ }_{D, r}$ and $\ell^{+}{ }_{D, t}$ in which case $\ell^{+} D$ can be taken as the average value of the three experimental values $\ell^{+} D, f, \ell^{+} D, r$ and $\ell^{+} D, t$ :

$$
\ell_{D}^{+}=\left(\ell_{D, f}^{+}+\ell_{D, r}^{+}+\ell_{D, t}^{+}\right) / 3
$$

In complete analogy with the previous case, solving Eq.(16) for $\ell$, one takes: 


$$
\begin{aligned}
& \left(\ell_{D, f, r, t}^{-}\right)^{2}-\left(2\left|\frac{C_{f, r, t}}{\lambda_{m, f, r, t}}\right| \frac{1-\varrho}{K R}\right)^{2 / 3}\left(1+2 \frac{C_{f, r, t}}{\lambda_{m, f, r, t}}\left|\frac{\lambda_{m, f, r, t}}{C_{f, r, t}}\right|\right)\left(\ell_{D, f, r, t}^{-}\right)^{4 / 3}+ \\
& \left(2\left|\frac{C_{f, r, t}}{\lambda_{m, f, r, t}}\right| \frac{1-\varrho}{K R}\right)^{4 / 3}\left(1+2 \frac{\lambda_{m, f, r, t}}{C_{f, r, t}}\left|\frac{C_{f, r, t}}{\lambda_{m, f, r, t}}\right|\right)\left(\ell_{D, f, r, t}^{-}\right)^{2 / 3}-\left(2\left|\frac{C_{f, r, t}}{\lambda_{m, f, r, t}}\right| \frac{1-\varrho}{K R}\right)^{2}-\left(\frac{D_{f, r, t}^{-}}{2 \lambda_{m, f, r, t}}\right)^{2}=0
\end{aligned}
$$

which, after setting:

$$
\ell_{D, f, r, t}^{-}=\left(m_{f, r, t}^{-}\right)^{3 / 2}
$$

becomes:

$$
\begin{aligned}
& \left(m_{f, r, t}^{-}\right)^{3}-\left(2\left|\frac{C_{f, r, t}}{\lambda_{m, f, r, t}}\right| \frac{1-\varrho}{K R}\right)^{2 / 3}\left(1+2 \frac{C_{f, r, t}}{\lambda_{m, f, r, t}}\left|\frac{\lambda_{m, f, r, t}}{C_{f, r, t}}\right|\right)\left(m_{f, r, t}^{-}\right)^{2}+ \\
& \left(2\left|\frac{C_{f, r, t}}{\lambda_{m, f, r, t}}\right| \frac{1-\varrho}{K R}\right)^{4 / 3}\left(1+2 \frac{\lambda_{m, f, r, t}}{C_{f, r, t}}\left|\frac{C_{f, r, t}}{\lambda_{m, f, r, t}}\right|\right) m_{f, r, t}^{-}-\left(2\left|\frac{C_{f, r, t}}{\lambda_{m, f, r, t}}\right| \frac{1-\varrho}{K R}\right)^{2}-\left(\frac{D_{f, r, t}^{-}}{2 \lambda_{m, f, r, t}}\right)^{2}=0
\end{aligned}
$$

Eq.(24) has one real solution which upon being introduced in Eq.(23) provides $\ell^{-} D_{, f, r, t}$ as:

$$
\begin{aligned}
& \ell_{D, f, r, t}^{-}=\left\{\frac{1}{3}\left(2\left|\frac{C_{f, r, t}}{\lambda_{m, f, r, t}}\right| \frac{1-\varrho}{K R}\right)^{2 / 3}\left(1+2 \frac{C_{f, r, t}}{\lambda_{m, f, r, t}}\left|\frac{\lambda_{m, f, r, t}}{C_{f, r, t}}\right|\right)+\right. \\
& \left\{( 2 | \frac { C _ { f , r , t } } { \lambda _ { m , f , r , t } } | \frac { 1 - \varrho } { K R } ) ^ { 2 } \left\{\left(1+2 \frac{C_{f, r, t}}{\lambda_{m, f, r, t}}\left|\frac{\lambda_{m, f, r, t}}{C_{f, r, t}}\right|\right)\left[\frac{1}{27}\left(1+2 \frac{C_{f, r, t}}{\lambda_{m, f, r, t}}\left|\frac{\lambda_{m, f, r, t}}{C_{f, r, t}}\right|\right)^{2}-\frac{1}{6}\left(1+2 \frac{\lambda_{m, f, r, t}}{C_{f, r, t}}\left|\frac{C_{f, r, t}}{\lambda_{m, f, r, t}}\right|\right)\right]+\right.\right. \\
& \left.\frac{1}{2}\left[1+\left(\frac{K R D_{f, r, t}^{-}}{4(1-\varrho) C_{f, r, t}}\right)^{2}\right]\right\}+\left\{\left(2\left|\frac{C_{f, r, t}}{\lambda_{m, f, r, t}}\right| \frac{1-\varrho}{K R}\right)^{4}\left[\frac{1}{3}\left(1+2 \frac{\lambda_{m, f, r, t}}{C_{f, r, t}}\left|\frac{C_{f, r, t}}{\lambda_{m, f, r, t}}\right|\right)-\frac{1}{9}\left(1+2 \frac{C_{f, r, t}}{\lambda_{m, f, r, t}}\left|\frac{\lambda_{m, f, r, t}}{C_{f, r, t}}\right|\right)^{2}\right]^{3}+\right. \\
& \left(2\left|\frac{C_{f, r, t}}{\lambda_{m, f, r, t}}\right| \frac{1-\varrho}{K R}\right)^{4}\left\{\left(1+2 \frac{C_{f, r, t}}{\lambda_{m, f, r, t}}\left|\frac{\lambda_{m, f, r, t}}{C_{f, r, t}}\right|\right)\left[\frac{1}{27}\left(1+2 \frac{C_{f, r, t}}{\lambda_{m, f, r, t}}\left|\frac{\lambda_{m, f, r, t}}{C_{f, r, t}}\right|\right)^{2}-\frac{1}{6}\left(1+2 \frac{\lambda_{m, f, r, t}}{C_{f, r, t}}\left|\frac{C_{f, r, t}}{\lambda_{m, f, r, t}}\right|\right)\right]+\right. \\
& \left.\left.\left.\frac{1}{2}\left[1+\left(\frac{K R D_{f, r, t}^{-}}{4(1-\varrho) C_{f, r, t}}\right)^{2}\right]\right\}^{2}\right\}^{1 / 2}\right\}^{1 / 3}+ \\
& \left\{( 2 | \frac { C _ { f , r , t } } { \lambda _ { m , f , r , t } } | \frac { 1 - \varrho } { K R } ) ^ { 2 } \left\{\left(1+2 \frac{C_{f, r, t}}{\lambda_{m, f, r, t}}\left|\frac{\lambda_{m, f, r, t}}{C_{f, r, t}}\right|\right)\left[\frac{1}{27}\left(1+2 \frac{C_{f, r, t}}{\lambda_{m, f, r, t}}\left|\frac{\lambda_{m, f, r, t}}{C_{f, r, t}}\right|\right)^{2}-\frac{1}{6}\left(1+2 \frac{\lambda_{m, f, r, t}}{C_{f, r, t}}\left|\frac{C_{f, r, t}}{\lambda_{m, f, r, t}}\right|\right)\right]+\right.\right. \\
& \left.\frac{1}{2}\left[1+\left(\frac{K R D_{f, r, t}^{-}}{4(1-\varrho) C_{f, r, t}}\right)^{2}\right]\right\}-\left\{\left(2\left|\frac{C_{f, r, t}}{\lambda_{m, f, r, t}}\right| \frac{1-\varrho}{K R}\right)^{4}\left[\frac{1}{3}\left(1+2 \frac{\lambda_{m, f, r, t}}{C_{f, r, t}}\left|\frac{C_{f, r, t}}{\lambda_{m, f, r, t}}\right|\right)-\frac{1}{9}\left(1+2 \frac{C_{f, r, t}}{\lambda_{m, f, r, t}}\left|\frac{\lambda_{m, f, r, t}}{C_{f, r, t}}\right|\right)^{2}\right]^{3}+\right.
\end{aligned}
$$




$$
\begin{aligned}
& +\left(2\left|\frac{C_{f, r, t}}{\lambda_{m, f, r, t}}\right| \frac{1-\varrho}{K R}\right)^{4}\left\{\left(1+2 \frac{C_{f, r, t}}{\lambda_{m, f, r, t}}\left|\frac{\lambda_{m, f, r, t}}{C_{f, r, t}}\right|\right)\left[\frac{1}{27}\left(1+2 \frac{C_{f, r, t}}{\lambda_{m, f, r, t}}\left|\frac{\lambda_{m, f, r, t}}{C_{f, r, t}}\right|\right)^{2}-\frac{1}{6}\left(1+2 \frac{\lambda_{m, f, r, t}}{C_{f, r, t}} \mid \frac{C_{f, r, t}}{\lambda_{m, f, r, t}}\right)\right]+\right. \\
& \left.\left.\left.\left.\left.\frac{1}{2}\left[1+\left(\frac{K R D_{f, r, t}^{-}}{4(1-\varrho) C_{f, r, t}}\right)^{2}\right]\right\}\right\}^{2}\right\}^{1 / 2}\right\}^{1 / 3}\right\}^{3 / 2}
\end{aligned}
$$

and

$$
\ell_{D}^{-}=\left(\ell_{D, f}^{-}+\ell_{D, r}^{-}+\ell_{D, t}^{-}\right) / 3
$$

Eventually, supposing that all $D_{f, r, t}^{+}$and $D_{f, r, t}$ are measurable on caustics' photos, the respective "experimental" $\ell_{D}$ will be obtained as the average value of $\ell^{+} D$ and $\ell^{-} D$ :

$$
\ell_{D}=\left(\ell_{D}^{+}+\ell_{D}^{-}\right) / 2
$$

Clearly, in case of simple caustics where only $D_{f, 5, t}^{+}$exists, the "experimental" $\ell_{D}$ will coincide with $\ell^{+} D$ of Eq.(21). It should be mentioned however, that usually in practice it is not feasible to measure all of the $D^{ \pm}{ }_{f, r}$ on caustic's photos due to the partially overlapped reflected caustics (front and rear) and thus not all of the experimental $\ell^{ \pm} D_{D, f, r, t}$ can be calculated from Eqs. $(20,25)$; in that case the final "experimental" $\ell_{D}$ will be the average value of those $\ell^{ \pm} D_{D, f, n, t}$ that could be calculated.

Obtaining the contact length from the elevations of the extreme points of the general contact caustic curves

In the more general case of double caustics, the elevations $H_{f, r, t}^{ \pm}$of the end points $E_{f f, r t}^{ \pm} L_{f f, r}^{ \pm}$of caustics (Fig.7), will be defined by the aid of the second of Eqs. (13), as:

$$
\begin{aligned}
& H_{f, r, t}^{+}=Y_{f, r, t}^{+}(\theta=0)=C_{f, r, t}\left[2 \frac{1-\varrho}{K R}-\left(2 \frac{1-\varrho}{K R \ell}\right)^{2 / 3}\left|\frac{\lambda_{m, f, r, t}}{C_{f, r, t}}\right|^{1 / 3} \sqrt{1+\left(2\left|\frac{C_{f, r, t}}{\lambda_{m, f, r, t}}\right| \frac{1-\varrho}{K R \ell}\right)^{2 / 3}}\right] \\
& H_{f, r, t}^{-}=Y_{f, r, t}^{-}(\theta=0)=2 \frac{1-\varrho}{K R} C_{f, r, t}
\end{aligned}
$$

Eq.(29) does not include $\ell$ and it cannot be used directly to obtain $\ell$, however, it can be proven very useful in specifying the location of $X_{f, t}$-axes (see below) which is the basic requirement for measuring the elevations $H_{f, r, t}^{+}$in experimental caustic's photos. Thus, in the case of double caustics, only Eq.(28) can be directly used to obtain $\ell$ (from only the elevations $H_{f, r, t}^{+}$. In this context, solving Eq.(28) for $\ell$ yields:

$$
\ell_{H, f, r, t}=2\left|\frac{C_{f, r, t}}{\lambda_{m, f, r, t}}\right| \frac{1-\varrho}{K R}\left\{\left[\frac{K R H_{f, r, t}^{+}}{2(1-\varrho) C_{f, r, t}}-1\right]^{2}-1\right\}^{3 / 2}
$$

which with few inessential modifications (for the sake of generality) is the second formula obtained in [26] for determining $\ell$. As previously, writing $\ell_{H, f, n}$, instead of just $\ell$ (superscript + has now been omitted for obvious reasons), is to indicate that now $\ell$ is obtained from the elevations $H_{f, r, t}^{+}$and to stress the distinction that should be made among the experimental values $\ell_{H, f}, \ell_{H, r}$ and $\ell_{H, t}$ with respect to each other and with respect to the theoretical $\ell$ of Eqs. (1), due to reasonable slight differences expected between theoretical and experimental $\mathrm{H}_{f}^{+} \mathrm{H}^{+}{ }_{r}$ and $\mathrm{H}^{+}{ }_{t}$-values Thus, supposing that $\mathrm{H}^{+}{ }_{f}, \mathrm{H}^{+}{ }_{r}$ and $\mathrm{H}^{+}{ }_{t}$ have been measured on caustics' photos then introducing these values in Eq.(30), $\ell_{H, f}, \ell_{H, r}$ and $\ell_{H, t}$ are calculated; the final "experimental" $\ell_{H}$ will be their average value:

$$
\ell_{H}=\left(\ell_{H, f}+\ell_{H, r}+\ell_{H, t}\right) / 3
$$


Obviously, obtaining $\ell_{H}$ in the case of single caustics presents no difference from the case of double ones, since only the elevations $H_{f, r, t}^{+}$are used in both cases, that is, Eqs. $(30,31)$ hold also true in the case of single caustics.

In conclusion, having $\ell_{D}$ from Eq.(27) (see previous paragraph) and $\ell_{H}$ from Eq.(31), the final "experimental” $\ell_{\exp }$ can be quite accurately defined as:

$$
\ell_{\exp }=\left(\ell_{D}+\ell_{H}\right) / 2
$$

There of course remains the problem of specifying the $X_{f, t}$-axes positions on reflected and transmitted caustics' photos in order that $H_{f, r, t}^{+}$can be measured.

Specifying the position of $X_{f, t}$-axes on reflected and transmitted caustics' photos

To measure $H_{f, r}^{+}$(required in Eq.(30) to obtain $\ell_{H, f, r, t}$ ), locating the positions of $X_{f, t}$-axes on caustics' photos is sine qua non. In this context, two ways are next proposed to locate $X_{f, t}$-axes. Namely, in the case of double caustics, it has been seen that Eq.(29) provides the elevations $H_{f, r, t}^{-}$of the innermost end points $E_{f, r, t}^{-}$or $L_{f, r, t}$ (Fig.7) of the double caustics independently of $\ell_{H, f, r, t}$; actually, $H_{-f, r, t}$ depend only on the experimental set-up. Thus, detecting on caustics' photos the innermost end points $E_{f, r, t}^{-}$(or $L_{f, r, t}$ ) of double caustics and then drawing from these points normally the respective $\ell_{H, f, r, t}$ independent elevations $H_{f, r, t}, X_{f, t}$-axes are automatically obtained on the photos independently of $\ell_{H}$, and thus they can be safely considered as a basis in obtaining $\ell_{H, f, r, t,}$. Indeed, having $X_{f, t}$-axes on caustics' photos, measuring $H_{f, r, t}^{+}$and in turn obtaining $\ell_{H, f, r, t}$ and $\ell_{H}$ from Eqs. (30) and (31) is a trivial procedure. Obviously, the higher the number of detectable points $E_{f, r, t}$ (or/and $L_{f, r, t}$ ) on the photos is, the more accurate the location of $X_{f, t}$-axes on caustics' photos will be. Clearly, that simple approach does not apply to single caustics since in that case there are no any innermost ends $E_{f, r, t}^{-}{ }^{-} L_{f, r, t}{ }^{-}$ However, there is an alternative approach to specify $X_{f, t}$-axes on caustics' photos graphically, at least in a first approximation, applicable to both cases (double and single caustics). Namely, it is seen that $X_{f, t}$-axes pass approximately from the intersection of the lines $\mathrm{E}^{+}{ }_{t} \mathrm{~L}_{f}$ or $\mathrm{E}^{+}{ }_{f} \mathrm{~L}_{t}^{+}$with the vertical symmetry axes of caustics, i.e., the $Y_{f, t}$-axes either in the case of double (Fig.8a) or single (Fig.8b) caustics.
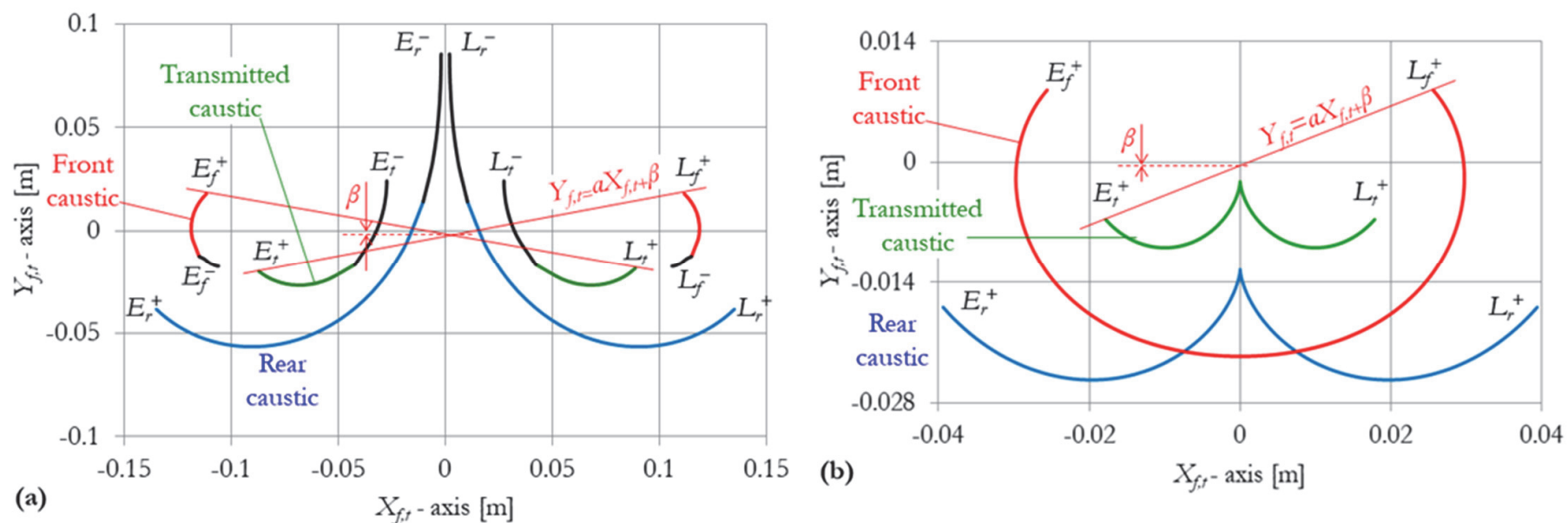

Figure 8: Obtaining graphically the $X_{f t}$-axes, (a) in the case of double and (b) in the case of single caustics.

Of course, the points $L_{f}^{+}$and $\mathrm{E}_{f}^{+}$are located on the front screen while the points $\mathrm{E}^{+}{ }_{t}$ and $\mathrm{L}_{t}^{+}$are located on the rear one; thus, in order that these points can be combined to form the above lines, the points $E^{+}{ }_{t}, L^{+}{ }_{t}\left(E^{+} f, L_{f}^{+}\right)$from the rear (front) photos should be properly specified on the front (rear) photos containing the points $E_{f}^{+}, L_{f}^{+}\left(E^{+} t_{t} L_{t}^{+}\right)$. For example, the equation of the line $E^{+}{ }_{t} L_{f}^{+}$(in both cases of Fig.(8a,b), which is of the form $Y_{f, t}=a X_{f, t}+\beta$, with $a$ the slope, and $\beta$ the ordinate of the point the line $\mathrm{E}^{+}{ }_{t} \mathrm{~L}^{+}$intersects $Y_{f, t}$-axis, is given as:

$$
Y_{f, t}=\underbrace{\frac{Y_{L_{f}^{+}}^{+}-Y_{E_{t}^{+}}^{+}}{X_{L_{f}^{+}}^{+}-X_{E_{t}^{+}}^{+}}}_{a} X_{f, t}+\underbrace{Y_{E_{t}^{+}}^{+}-\frac{Y_{L_{f}^{+}}^{+}-Y_{E_{t}^{+}}^{+}}{X_{L_{f}^{+}}^{+}-X_{E_{t}^{+}}^{+}} X_{E_{t}^{+}}^{+}}_{\beta}=\underbrace{\frac{Y_{L_{f}^{+}}^{+}-Y_{E_{t}^{+}}^{+}}{X_{L_{f}^{+}}^{+}-X_{E_{t}^{+}}^{+}}}_{a} X_{f, t}+\underbrace{Y_{L_{f}^{+}}^{+}-\frac{Y_{L_{f}^{+}}^{+}-Y_{E_{t}^{+}}^{+}}{X_{L_{f}^{+}}^{+}-X_{E_{t}^{+}}^{+}} X_{L_{f}^{+}}^{+}}_{\beta}
$$

where 


$$
\begin{aligned}
& X_{E_{t}^{+}}^{+}=X_{t}^{+}(\theta=-\pi) \underbrace{=}_{\mathrm{Eq} \cdot(13(\mathrm{a}))}-\lambda_{m, t} \ell \sqrt{1+\left(2\left|\frac{C_{t}}{\lambda_{m, t}}\right| \frac{1-\varrho}{K R \ell}\right)^{2 / 3}} \\
& Y_{E_{t}^{+}}^{+}=Y_{t}^{+}(\theta=-\pi) \underbrace{=}_{\text {Eq. }(13(\mathrm{~b}))} C_{t}\left[2 \frac{1-\varrho}{K R}-\left(2 \frac{1-\varrho}{K R \ell}\right)^{2 / 3}\left|\frac{\lambda_{m, t}}{C_{t}}\right|^{1 / 3} \ell \sqrt{1+\left(2\left|\frac{C_{t}}{\lambda_{m, t}}\right| \frac{1-\varrho}{K R \ell}\right)^{2 / 3}}\right] \\
& X_{L_{f}^{+}}^{+}=X_{f}^{+}(\theta=0) \underbrace{=}_{\text {Eq. }(13(\mathrm{a}))} \lambda_{m, f} \ell \sqrt{1+\left(2\left|\frac{C_{f}}{\lambda_{m, f}}\right| \frac{1-\varrho}{K R \ell}\right)^{2 / 3}} \\
& Y_{L_{f}^{+}}^{+}=Y_{f}^{+}(\theta=0) \underbrace{=}_{\text {Eq. }(13(\mathrm{~b}))} C_{f}\left[2 \frac{1-\varrho}{K R}-\left(2 \frac{1-\varrho}{K R \ell}\right)^{2 / 3}\left|\frac{\lambda_{m, f}}{C_{f}}\right|^{1 / 3} \ell \sqrt{1+\left(2\left|\frac{C_{f}}{\lambda_{m, f}}\right| \frac{1-\varrho}{K R \ell}\right)^{2 / 3}}\right]
\end{aligned}
$$

Usually, $\beta$ is too small so that in a first approximation, it can be considered zero, in which case the line $E^{+}{ }_{t} L^{+}{ }_{f}$ passes from the origin thus defining the $X_{f, t}$-axes. So having $X_{f, t}$-axes and any of the outermost end points $E_{f, r, t}^{+}\left(\right.$or $\left.L_{f, r, t}^{+}\right)$on caustics' photos, $H_{f, r, t}^{+}$can be directly measured and in turn $\ell_{H}$ is obtained by the aid of Eqs. (30) and (31).

\section{EXPERIMENTAL PROCEDURE}

A series of experiments were implemented to assess the efficiency of the previously provided general formulae and approach for obtaining the contact length in the case of double caustics. In this context, taking advantage of the previous analysis with regard to the conditions for the development of double curves, the imposed loading and features of the optical set-up were suitably chosen so that double caustics can occur, trying at the same time not to exceed the linearity limit of the material of the specimen. It is to be mentioned that the experimental protocol described below is only a first step of an ongoing research the complete results of which will be presented in a future work.

\section{The experimental arrangement}

The experimental set-up is shown in the sketch of Fig.2. It was consisted of a He-Ne laser tube emitting a narrow beam of red coherent light (of a wave length about $600 \mathrm{~nm}$ ), refined and spread out as passing through a pin hole of $50 \mu \mathrm{m}$ mounted before the output coupler of the laser. The outspread light is received by a first lens transforming it into a parallel wide coherent light beam that converges again as passing through the second lens. Moving the second lens back and forth its focus point may be located at the desired distance $Z_{i}$ in front of or behind the cylindrical specimen, resulting to a divergent or convergent incident light beam respectively and defining at one's convenience the magnitude and sigh of the magnification factors $\lambda_{m, f, s, t}$ (Eqs. (3)). A semi-reflector, placed between the second lens and the specimen at an angle $45^{\circ}$ with respect the specimen's cross-section, facilitates receiving the reflected light on the front reference screen; in that case $Z_{o, f}$ is the total distance: specimen's middle cross-section - semi-reflector - front screen. Light passing through the transparent specimen is received on the rear reference screen, placed directly behind and parallel to the specimen's middle cross-section at a distance $Z_{o, r}$.

The specimen was fixed within the jaws of the ISRM apparatus for the implementation of the Brazilian-disc test which was mounted at an electromechanical INSTRON 1125 loading frame of $50 \mathrm{kN}$ capacity. The movable traverse of the loading frame moved downwards, at a rate of $0.1 \mathrm{~mm} / \mathrm{min}$, compressing the upper jaw of the ISRM apparatus, and in turn the cylindrical specimen. Normality of the loading axis was ensured by a semi-spherical head interposed between the traverse of the loading frame and the jaw bearing on its upper face a suitably perforated hollow cavity fitting the semispherical head. The lower jaw was placed on a $50 \mathrm{kN}$ compression load cell calibrated with a verified Wykeham Farrance compression ring of $10.62 \mathrm{~N}$ sensitivity. The cell exhibited a linear behavior for the whole loading range with a deviation less than $0.2 \%$ while the displacement rate was also calibrated by a High Mag micrometric calibrator, exhibiting also a linear behavior with a deviation less than $0.4 \%$.

Regarding the specimen, it was a cylinder of radius $R=5 \mathrm{~cm}$ and thickness $t=1 \mathrm{~cm}$, made of PMMA with Young's modulus $E=3.20 \mathrm{GPa}$ and a Poisson's ratio $v=0.38$. The specific values of the elastic constants of the material were determined 
through standardized uniaxial tests in rectangular specimens of dimensions $(25 \times 2 \times 0.2) \mathrm{cm}$, of the same batch of the material, resulting to a stress-optical constant $c_{f}=1.19 \times 10^{-7} \mathrm{~m}^{2} / \mathrm{kN}$ (first of Eqs. (5)). In addition, for the particular batch of the material, $k$ was estimated equal to 1.50 , resulting to a $c_{r}=1.79 \times 10^{-7} \mathrm{~m}^{2} / \mathrm{kN}$ (second of Eqs. (5)).

\section{The experimental protocol}

In this first series of experiments the parameters of the experimental/optical set-up were properly chosen so that double transmitted and single reflected caustics will be obtained. The choice of receiving the transmitted double caustics instead of the reflected ones is to be attributed to the fact that the revealing for example of the double front reflected caustics would require a shorter distance $Z_{i}$ between the focus point of the second lens and the specimen or a high level of the externally applied load. In the first case, as the focus of the impinging divergent light is too close to the specimen, so that the area illuminated is very confined, quality of caustics is not very good with secondary optical phenomena, difficult to describe, shadowing in most of the experimental attempts undertaken the results. In the second case, the load would be required to obtain the double reflected front curves would be, unfortunately, for the particular material, too high to suffice the material would remain in its linearly elastic state, a crucial assumption in the deduction of the analytic formulae (see also the discussion in the concluding section). In this context, and for a $k=1.5$ for the particular batch of the material (second of Eqs. (5)), the following settings were made: $Z_{0, f}=1.0 \mathrm{~m}, Z_{0, t}=0.2$ and $Z_{i}=0.2 \mathrm{~m}$. In addition, the load level was quasi-statically increased from zero up to $P_{\text {frame }}=3 \mathrm{kN}$.

During loading, a series of photos of caustics taken from both the front and rear screens were obtained (Fig.9). Namely, in Figs. 9(a1, a2), 9(b1, b2) and 9(c1, c2), the photos of reflected and transmitted caustics are shown for three specific loading levels, $P_{\text {frame }}=1 \mathrm{kN}, 2.5 \mathrm{kN}$ and $3 \mathrm{kN}$, respectively. Then, by the aid of these experimental results and the previous analytic formulae, the contact length was obtained from the expressions involving the spans $D^{ \pm} f, t$, of the caustics that were available/measurable on the photos of experimental caustics (the formulae involving the elevations $H_{f, r, t}^{ \pm}$were not used here). Actually, the experimental results, viz., the distances $D^{ \pm}{ }_{f, r}$ used for estimating the contact length, were exclusively pumped out of Figs. 9(b1) and 9(b2). In the other figures, apart from presenting the gradual evolution of caustics upon load increment, relevant information is given for clarity, as for example the dimensions of the 2- and 1-cent of euro coins attached to the front and rear screens, respectively, for the necessary measurements to be feasible.

Namely, from Fig.9(b1) it is found that $D^{+}{ }_{f}=7 \mathrm{~cm}$ and $D^{+}{ }_{r}=8.5 \mathrm{~cm}$, while from Fig.9(b2) it is seen that $D^{+}{ }_{t}=2.4 \mathrm{~cm}$ and $D_{-}=0.3 \mathrm{~cm}$. Then introducing the values measured, $D^{+}{ }_{f}=7 \mathrm{~cm}, D^{+}{ }_{r}=8.5 \mathrm{~cm}$ and $D^{+}{ }_{t}=2.4 \mathrm{~cm}$ in Eq.(20), the experimental values for $\ell_{D, f}^{+} \ell^{+}{ }_{D, r}$ and $\ell^{+}{ }_{D, t}$ are calculated as $\ell^{+}{ }_{D, f}=0.41 \mathrm{~cm}, \ell^{+}{ }_{D, r}=0.39 \mathrm{~cm}$ and $\ell^{+}{ }_{D, t}=0.46 \mathrm{~cm}$, respectively, whence by Eq.(21) one takes the experimental value $\ell^{+}{ }_{D}=0.42 \mathrm{~cm}$. On the other hand, introducing the value $D^{-}{ }_{t}=0.3 \mathrm{~cm}$ in Eq.(25), the experimental value for $\ell^{-}{ }_{D, t}$ is calculated as $\ell^{-}{ }_{D, t}=0.43$, whence Eq.(26) obviously yields again $\ell^{-}{ }_{D}=0.43 \mathrm{~cm}$ (since the transmitted caustic is here the only double one). Finally, combining $\ell^{+} D_{D}=0.42 \mathrm{~cm}$ and $\ell^{-}{ }_{D}=0.43 \mathrm{~cm}$, Eq. (27) provides here the experimental value $\ell_{D}=0.425 \mathrm{~cm}$ for the half contact length, which is in a quite good agreement with the respective theoretical value $\ell=0.388 \mathrm{~cm}$, provided by the second expression of Eqs. (1), for the particular data.

\section{CONCLUSIONS}

I $\mathrm{n}$ the present paper, an effort was undertaken to describe the nature of double initial and caustic curves, generated in the contact region realized between two cylindrical bodies when compressed against each other, and then based on the above description to extend/complete existing formulae [26] for obtaining the contact length. As it is shown, single curves are actually a particular case of double ones with the latter corresponding to the whole range of possible solutions of Eq.(7) that provides the radius of the initial curve. Namely, Eq.(7) admits in general three solutions, a real, a complex and a purely imaginary one (Fig.4b), where of course only the real one can be justified to stand as the radius of the initial curve. It is seen that in that case the initial curve splits into two parts, the left and the right, each one of which further consists of two branches the outermost and the innermost (Fig.4c). In the limiting case where there is only a real solution to Eq.(7), the initial curve consists of one part with one branch, which is the usual case of single initial and in turn caustic curve. In the same direction, it was shown that under certain assumptions six independent parameters can be distinguished influencing the occurrence of double or single curves, viz., the load level, the material's compliance and various features of the optical set-up. In this context, it was shown that is not always an extended contact length responsible for the appearance of double curves; for example, a small distance $Z_{i}$ between the focus point of the last $\left(2^{\text {nd }}\right)$ lens and the specimen or a small distance $Z_{o, t}$ between the specimen's cross-section and the rear reference screen may lead to double curves even in the case of low load levels and small contact lengths. Namely, as it was seen from the experimental protocol, similar single and double curves exist simultaneously and it is up to the particular choice of the 
characteristics of the optical set-up for which of them (double or single) will materialize, thus, a big value for $Z_{o, t}$ would reveal the single transmitted caustic while the small one adopted in the experiment revealed the double one (see also the relevant Fig.5f). Under the above conclusions, the existing formulae [26] for obtaining the contact length by the aid of the contact caustics are completed by including the case of double caustics, and it is shown that in the same way the spans $D_{f, r, t}^{+}$and elevations $H_{f, r, t,}^{+}$of single caustics are used to estimate the half contact length $\ell$, the values $D^{-}{ }_{f, r, t}$ and $H_{f, r, t}\left(F_{i g}\right.$. 7) can be similarly used to obtain $\ell$ in the case of double caustics. In this context, the explicit expressions for both $\ell^{+} D, f, r, t$ (Eq.(20)) and $\ell^{-}{ }_{D, f, r, t}$ (Eq.(25)) are here given for the first time, i.e., the half contact length as obtained from the caustic curves' spans $D_{f, r, t}^{+}$in the case of either single or double caustics, and $D_{-f, r, t}$ in the case of only double caustics, respectively.

(a1)

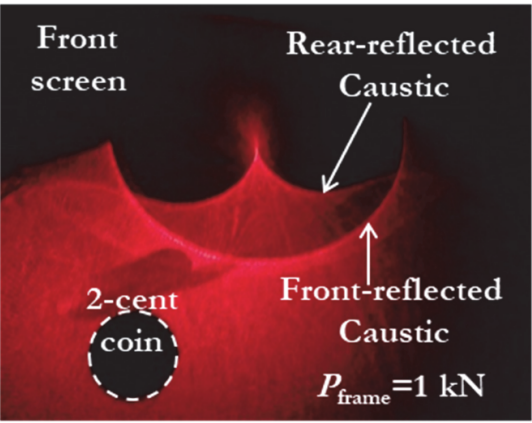

(b1)

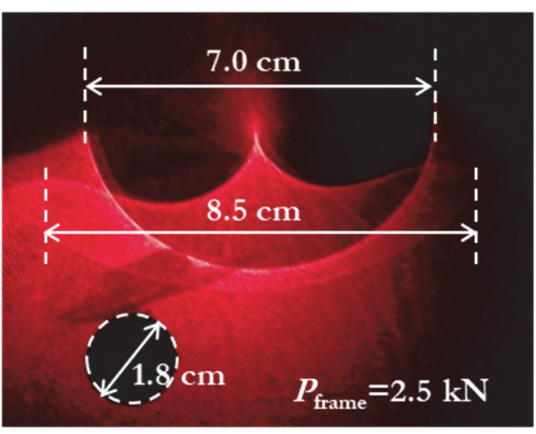

(c1)

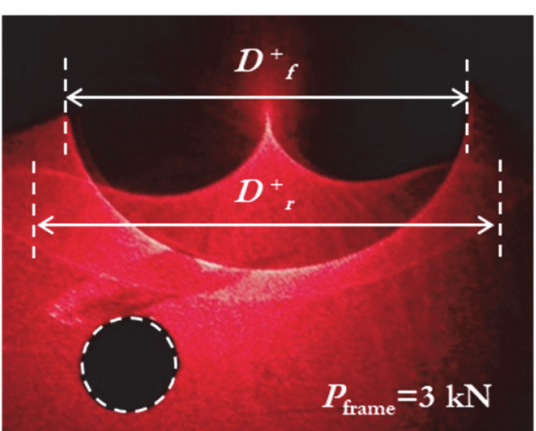

(a2)

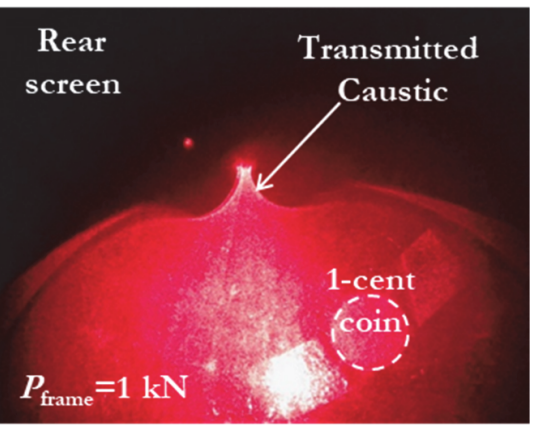

(b2)

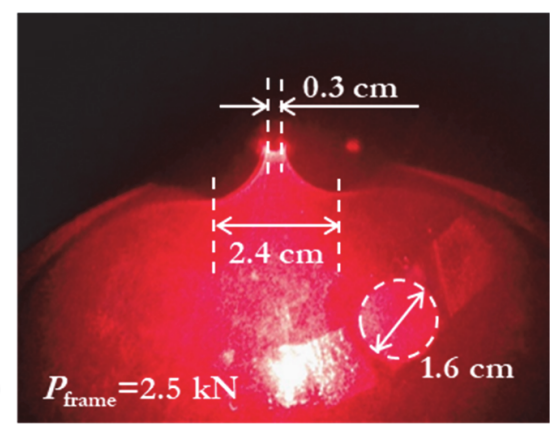

(c2)

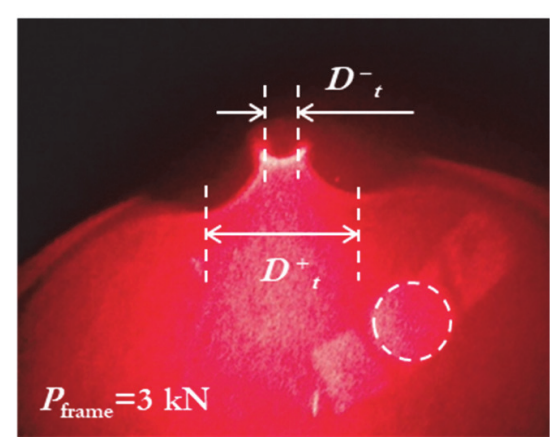

Figure 9: Experimental caustics for obtaining the contact length: Front and rear single reflected caustics for (a1) $P_{\text {frame }}=1 \mathrm{kN},(\mathrm{b} 1)$ $P_{\text {frame }}=2.5 \mathrm{kN}$ and (c1) $P_{\text {frame }}=3 \mathrm{kN}$; Transmitted double caustics for (a2) $P_{\text {frame }}=1 \mathrm{kN}$, (b2) $P_{\text {frame }}=2.5 \mathrm{kN}$ and (c2) $P_{\text {frame }}=3 \mathrm{kN}$

In addition, two ways for specifying the $X_{f, t}$-axes on experimental caustics' photos are given, in the case of single and double curves, necessary for applying the existing linear formula [26] for obtaining $\ell_{H, f, r, t}$, i.e., the half contact length $\ell$ through the respective elevations.

To assess the formulae obtained for the general (double) caustics, an experimental protocol concerning transparent thin circular discs made of PMMA, squeezed in the ISRM's apparatus for the standardized implementation of the Braziliandisc test, was carried out. Though it is obvious that the experiments conducted here could not cover the full range of the experimental investigation required for describing the whole subject and revealing all the features regarding the formation and exploitation of double caustics in praxis, at least they showed, at a very first stage, the efficiency of the method and the general formulae.

It is to be mentioned, however, that caustics apart from being a highly sensitive and accurate method concerning the results drawn, it is also very sensitive regarding the preparation and implementation of the experiment, and should be used 
with caution. For example, the photos of caustics in Figs. 9(c1) and 9(c2), though they seem more representative and perhaps more adequate than those of Figs. 9(b1) and 9(b2), are actually refer to the case the material has enter plasticity, in the contact region, so that any measurements should not be so simply used in obtaining the contact length (this is readily verified by unloading and reloading the specimen whence it is seen that the new contact caustics are by no means of an elastic nature).

Finally, it should be noticed that the general formulae including double and single caustics obviously refer also to opaque materials as particular cases of the transparent ones, provided their faces are properly polished. The latter could be of crucial importance, especially for specimens made of marble [50] and natural building stones [51], the mechanical response of which is usually studied using the Brazilian-disc test (due to insuperable difficulties encountered in the implementation of direct uniaxial tension tests, caused due to the unique nature of such materials [52-56]) employing either traditional or innovative experimental techniques [57-60].

\section{ACKNOWLEDGMENTS}

7 he author would like to express his deep gratitude to his Professor Kallimassiotis-Pazis, D. N., for his unreserved help and support in conducting the experimental protocol; his long experience in the application of optical methods in the investigation of the elastic equilibrium of various commercial materials were very crucial in obtaining reliable results and improve the quality of this paper.

\section{REFERENCES}

[1] Manogg, P. (1964). Anwendung der Schattenoptik zur Untersuchung des Zerreissvongangs von Platten, Dissertation, Freiburg, Germany.

[2] Manogg, P. (1964). Schattenoptische messung der spezifischen bruchenergie waehrend des bruchvorgangs bei plexiglas, in: Proceedings of the International Conference on Physics of Noncrystalline Solids, edited by J.A. Prins, Delft, The Netherlands, pp. 481-490.

[3] Manogg, P. (1966). Investigation of the rupture of a plexiglas plate by means of an optical method involving high speed filming of the shadows originating around holes drilled in the plate, Int. J. Fracture, 2(4), 604-613.

[4] Manogg, P. (1966). Die lichtablenkung durch eine elastisch beanspruchte platte und die schattenfiguren von kreisund risskerbe, Glastechnische Berichte, 39, pp. 323-329.

[5] Theocaris, P.S. (1970). Local yielding around a crack tip in plexiglas, J. Appl. Mech., 37(2), pp. 409-415.

[6] Theocaris, P.S. and Gdoutos, E.E. (1972). An optical method for determining opening-mode and edge sliding-mode stress intensity factors, J. Appl. Mech., 39(1), pp. 91-97.

[7] Kalthoff, J.F., Winkler, S. and Beinert, J. (1976). Dynamic stress intensity factors for arresting cracks in DCB specimens, Int. J. Fracture, 12(2), pp. 317-319.

[8] Theocaris, P.S. (1981). Elastic stress intensity factors evaluated by caustics, In: Mechanics of Fracture, edited by G. C. Sih, Sijthoff and Noordhoff, Amsterdam, The Netherlands, 7(3).

[9] Papadopoulos, G.A. (2011). New formula of experimental stress intensity factor evaluation by caustics, Int. J. Fracture, 171, pp. 79-84.

[10] Yazdanmehr, A. and Soltani, N. (2014). Evaluation of stress intensity factors of rounded V and U notches under mixed mode loading, using the experimental method of caustics, Theor. Appl. Fract. Mec., 74(1), pp. 79-85.

[11] Theocaris, P.S. and Andrianopoulos, N.P. (1981). Dynamic three-point bending of short beams studied by caustics, Int. J. Solids Struct., 17(7), pp. 707-715.

[12] Papadopoulos, G. A. (1990). Dynamics caustics and its applications, Opt. Laser Eng., 13(3-4), pp. 211-249.

[13] Georgiadis, H.G. and Papadopoulos, G.A. (1992). On the method of dynamic caustics in crack propagation experiments, Int. J. Fracture, 54(2), pp. R19-R22.

[14] Xiong C.Y., Yao, X.F., Fang, J. (1999). A study of dynamic caustics around running interface crack tip, Acta Mech. Sinica, 15(2), pp. 182-192.

[15] Yao, X.F., Xu, W., Jin, G.C., Yeh, H.Y. (2004). Low velocity impact study of laminate composites with mode I crack using dynamic optical caustics, J. Reinf. Plast. Comp., 23(17), pp. 1833-1844.

[16] Yao, X.F., Zhao, H.P., Yeh, H.Y. (2005). Dynamic caustic analysis of propagating mode II cracks in transversely isotropic material, J. Reinf. Plast. Comp., 24(6), pp. 657-667. 
[17] Yao, X.F., and Xu, W. (2011). Recent application of caustics on experimental dynamic fracture studies, Fatigue Fract. Eng. M., 34(6), pp. 448-459.

[18] Rosakis, A.J. and Freund, L.B. (1982). Optical measurements of the plastic strain concentration at a tip in a ductile steel plate, J. Eng. Mater. T., 104(2), pp. 115-125.

[19] Kikuchi, M. and Hamanaka, S. (1990). Evaluation of the J-integral by the caustics method, T. Jpn Soc. Mech. Eng. A, 56(532), pp. 2581-2587.

[20] Theocaris, P.S., Ioakimidis, N.I., (1979). On the determination of stress-optical constants by the method of reflected caustics, J. Phys. D Appl. Phys., 12(4), pp. 497-504.

[21] Younis, N.T. and Zachary, L.W. (1989). A new technique for the determination of stress-optical constants using the shadow spot method, Exp. Mech., 29(1), 75-79.

[22] Ioakimidis, N.I. and Theocaris, P.S. (1979). An optical method for the experimental determination of the Poisson ratio, Materialprüfung, 21, pp. 40-42.

[23] Theocaris, P.S. and Pazis, D.N. (1981). Some further properties of caustics useful in mechanical applications, Appl. Optics, 20(23), pp. 4009-4018.

[24] Theocaris, P.S. (1978). The load distribution in the generalized contact problem by caustics, In: Proceedings of the 3rd National Congress on Theoretical and Applied Mechanics, Varna, Bulgaria, 13-16 September 1977, Vol. III, pp.105-143.

[25] Theocaris, P.S. (1979). Experimental study of plane elastic contact problems by the pseudocaustics method, J. Mech. Phys. Solids, 27(1), pp. 15-32.

[26] Theocaris, P.S. and Stassinakis, C.A. (1978). The elastic contact of two disks by the method of caustics. Exp. Mech., 18(11), pp. 409-416.

[27] Semenski, D. (2003). Optical method of caustics - fulfilled experimental application to the contact problem, In: Proceedings of the XVII IMEKO World Congress Metrology in the 3rd Millennium, Dubrovnik, Croatia, June 22-27, 2003, pp. 1952-1955.

[28] Spitas, V., Papadopoulos, G.A., Spitas, C., Costopoulos, T. (2011). Experimental investigation of load sharing in multiple gear tooth contact using the stress-optical method of caustics, Strain, 47(1), pp. e227-e233.

[29] Raptis, K., Papadopoulos, G.A., Costopoulos, T.N., Tsolakis, A.D. (2011). Experimental study of load sharing in roller bearing contact by caustics and photoelasticity, Am. J. Eng. Appl. S., 4(2), pp. 294-300.

[30] Papadopoulos, G.A. and Pazis, D.N. (2003). The non-linear solution of the mixed-mode caustics in birefringent materials, Int. J. Fracture, 119(2), L35-L40.

[31] Yang, R.S., Yue, Z.W., Sun, Z.H., Xiao, T.S., Guo, D.M. (2009). Dynamic fracture behavior of rock under impact load using the caustics method, Min. Sci. Tech., 19(1), pp. 79-83.

[32] Gong, K., and Li, Z., (2008). Caustics method in dynamic fracture problem of orthotropic materials, Opt. Laser Eng., 46(8), pp. 614-619.

[33] Rossmanith, H.P., Knasmillner, R.E., Semenski, D. (1995). Crack-tip caustics in mechanically anisotropic materials, Exp. Mech., 35(1), pp. 31-35.

[34] Semenski, D. (1997). Method of caustics in fracture mechanics of mechanically anisotropic materials, Eng. Fract. Mech., 58(1-2), pp. 1-10.

[35] Semenski, D. and Jecić, S. (1999). Experimental caustics analysis in fracture mechanics of anisotropic materials, Exp. Mech., 39(3), pp. 177-183.

[36] Gao, G., Li, Z., Xu, J. (2011). Optical method of caustics applied in viscoelastic fracture analysis, Opt. Laser Eng., 49(5), pp. 632-639.

[37] Yao, X.F., Chen, J., Jin, G.C., Arakawa, K., Takahashi, K. (2004). Caustic analysis of stress singularities in orthotropic composite materials with mode-I crack, Compos. Sci. Technol., 64(7-8), pp. 917-924.

[38] Yao, X.F., Xu, W., Bai, S.L., Yeh, H.Y. (2008). Caustics analysis of the crack initiation and propagation of graded materials, Compos. Sci. Technol., 68(3-4), pp. 953-962.

[39] Theocaris, P.S., Ioakimidis, N.I. (1979). An improved method for the determination of mode I stress intensity factors by the experimental method of caustics, J. Strain Anal. Eng., 14(3), pp. 111-118.

[40] Rosakis, A.J. and Zehnder, A.T. (1985). On the method of caustics: an exact analysis based on geometrical optics, J. Elasticity 15(4), pp. 347-367.

[41] Wallhead, I.R., Güngör, S., Edwards, L. (1994). Optimisation of the optical method of caustics for the determination of stress intensity factors, Opt. Laser Eng., 20(2), pp. 109-133.

[42] Markides, Ch.F. and Kourkoulis, S.K. (2013). Revisiting the reflected caustics method: the accurate shape of the "initial curve", Eng. Transactions., 61(4), pp. 265-287. 
[43] Gdoutos, E.E. (2016). The optical method of caustics, Optics and Lasers in Engineering, 79, pp. 68-77

[44] Younis, N.T. (2011). Designing an optical mechanics experiment, W. Transactions Eng. Tech. Educ., 9(3), pp. 137144.

[45] Muskhelishvili, N.I. (1963). Some Basic Problems of the Mathematical Theory of Elasticity, Noordhoff, Groningen, The Netherlands.

[46] Markides, Ch.F. and Kourkoulis, S.K. (2016). The influence of jaw's curvature on the results of the Brazilian disc test, J. Rock Mech. Geotech. Eng., 8(2), pp. 127-146.

[47] ISRM (1978). Suggested methods for determining tensile strength of rock materials, Int. J. Rock Mech. Min., 15, pp. 99-103.

[48] Theocaris, P.S. (1981). Elastic stress intensity factors evaluated by caustics, in "Mechanics of Fracture, Vol. 7 Experimental evaluation of stress concentration and intensity factors. Useful methods and solutions to experimentalists in fracture mechanics", Sih, G.C. (ed.), Martinus Nijhoff, The Netherlands, pp. 189-252.

[49] Markides, Ch.F. (2018). Contact length in discs and rings by the reflected caustics, Procedia Struct. Integr., 10, pp. 163-170.

[50] Kourkoulis, S.K., Ganniari-Papageorgiou, E., Mentzini, M. (2010). Dionysos marble beams under bending: A contribution towards understanding the fracture of the Parthenon architraves, Eng. Geol., 115 (3-4), pp. 246-256, 2010.

[51] Kourkoulis, S.K., Ganniari-Papageorgiou, E. (2010). Experimental study of the size- and shape-effects of natural building stones, Constr. Build Mater., 24(5), pp. 803-810.

[52] Kourkoulis, S.K., Exadaktylos, G.E., Vardoulakis I. (1999). U-notched Dionysos-Pentelicon marble in three point bending: The effect of nonlinearity, anisotropy and microstructure, Int. J. Fracture, 98(3-4), pp. 369-392.

[53] Exadaktylos, G.E., Vardoulakis, I., Kourkoulis, S.K. (2001). Influence of nonlinearity and double elasticity on flexure of rock beams - I. Technical theory, Int. J. Solids Struct., 38(22-23), pp. 4091-4117.

[54] Exadaktylos, G.E., Vardoulakis, I., Kourkoulis, S.K. (2001). Influence of nonlinearity and double elasticity on flexure of rock beams - II. Characterization of Dionysos marble, Int. J. Solids Struct., 38(22-23), pp. 4119-4145.

[55] Vardoulakis, I., Exadaktylos, G.E., Kourkoulis, S.K. (1998). Bending of marble with intrinsic length scales: A gradient theory with surface energy and size effects, J. Phys. IV, 8, pp. 399-406.

[56] Kourkoulis, S.K., Prassianakis, I., Agioutantis, Z., Exadaktylos, G.E. (2006). Reliability assessment of the NDT results for the internal damage of marble specimens, Int. J. Mater. Prod. Tec., 26(1/2), pp. 35-56.

[57] Kourkoulis, S.K., Pasiou, E.D., Dakanali, I., Stavrakas, I., Triantis D. (2018). Notched marble plates under tension: Detecting pre-failure indicators and predicting entrance to the "critical stage", Fatigue Fract. Eng. M., 41(1), pp. 776786.

[58] Kourkoulis, S.K., Pasiou, E.D., Dakanali, I., Stavrakas, I., Triantis D. (2018). Notched marble plates under direct tension: Mechanical response and fracture, Constr. Build. Mater., 167, pp. 426-439.

[59] Kourkoulis, S.K., Dakanali, I., Pasiou, E.D., Stavrakas, I., Triantis D. (2017). Acoustic Emissions versus Pressure Stimulated Currents during bending of restored marble epistyles: Preliminary results, Fracture Struct. Integr., 41, pp. 536-551.

[60] Kourkoulis, S.K., Pasiou, E.D., Dakanali, I., Stavrakas, I., Triantis D. (2018). Mechanical response of notched marble beams under bending versus acoustic emissions and electric activity, J. Theor. Appl. Mech., 56 (2), pp. $523-547$. 